\title{
GRAPHS WITH SPECIFIED DEGREE DISTRIBUTIONS, SIMPLE EPIDEMICS, AND LOCAL VACCINATION STRATEGIES
}

\author{
TOM BRITTON, ${ }^{* * *}$ Stockholm University \\ SVANTE JANSON, ${ }^{* * *}$ Uppsala University \\ ANDERS MARTIN-LÖF, ${ }^{* * * * *}$ Stockholm University
}

\begin{abstract}
Consider a random graph, having a prespecified degree distribution $F$, but other than that being uniformly distributed, describing the social structure (friendship) in a large community. Suppose that one individual in the community is externally infected by an infectious disease and that the disease has its course by assuming that infected individuals infect their not yet infected friends independently with probability $p$. For this situation, we determine the values of $R_{0}$, the basic reproduction number, and $\tau_{0}$, the asymptotic final size in the case of a major outbreak. Furthermore, we examine some different local vaccination strategies, where individuals are chosen randomly and vaccinated, or friends of the selected individuals are vaccinated, prior to the introduction of the disease. For the studied vaccination strategies, we determine $R_{v}$, the reproduction number, and $\tau_{v}$, the asymptotic final proportion infected in the case of a major outbreak, after vaccinating a fraction $v$.
\end{abstract}

Keywords: Degree distribution; epidemic model; final size; limit theorem; networks; random graph; vaccination

2000 Mathematics Subject Classification: Primary 60C05; 05C80; 92D30

\section{Introduction}

Simple undirected random graphs can be used to describe the social network in a large community (e.g. [20]), where the vertices correspond to individuals and the edges correspond to some type of social structure, from now on referred to as friendships. Given such a graph, a model for the spread of the disease may be defined, where individuals at first are susceptible but may then become infected by a friend. An infected individual has the potential to spread the disease to his or her not yet infected friends before he or she recovers and becomes immune. The final outbreak, both its size and who becomes infected, depends on properties of the social graph as well as on properties of disease transmission. In order to prevent an outbreak it is possible to vaccinate, or immunise in some other way, individuals prior to the arrival of the disease. Who and how many individuals are to be vaccinated specifies the vaccination strategy.

In the present paper we study questions arising from such modeling. In particular, we consider random graphs where the degree distribution (i.e. the number of friends) follows some

Received 30 January 2007; revision received 26 October 2007.

* Postal address: Department of Mathematics, Stockholm University, SE-10691 Stockholm, Sweden.

** Email address: tom.britton@math.su.se

*** Postal address: Department of Mathematics, Uppsala University, PO Box 480, SE-75106 Uppsala, Sweden.

Email address: svante.janson@math.uu.se

**** Email address: andersml@math.su.se 
prespecified distribution $F$, typically having heavy tails, but where the random graph $G$ is otherwise uniformly distributed. The epidemic model is the simplest possible model for a susceptible-infectious-removed disease (e.g. [2, 11-18]). One randomly selected individual is initially externally infected. Any individual who becomes infected infects each of his or her not yet infected friends independently with probability $p$, and after that the individual recovers and becomes immune, a state called removed. For this graph and epidemic model, we study different vaccination strategies: the uniform strategy and the acquaintance strategy [7]. In both strategies individuals are chosen randomly from the community. In the uniform strategy the selected individuals are vaccinated, and in the acquaintance strategy a randomly chosen friend of the selected individual is vaccinated. Both vaccination strategies are local in the sense that the global social network need not be known in order to perform the strategy. We also study a vaccination strategy where, instead of selecting individuals at random, friendships are selected and one or two of the corresponding friends become vaccinated.

As the population size $n$ tends to $\infty$, we prove that the initial phase of the epidemic may be approximated by a suitable branching process. The largest eigenvalue of the branching process, often denoted by $R_{0}$ and called the basic reproduction number when applied to epidemics [2, 53-56], determines whether a major outbreak can occur or not: if $R_{0} \leq 1$, only minor outbreaks can occur, whereas if $R_{0}>1$, outbreaks of order $O(n)$ can also occur with positive probability. In case of a major outbreak the total number of individuals infected during the outbreak, the final size, is shown to satisfy a law of large numbers. The corresponding (random) proportion is shown to converge in probability to a deterministic limit $\tau_{0}$. Similar results are obtained when a vaccination strategy with vaccination coverage $v$ has been performed prior to disease introduction. In this situation, the strategy-specific reproduction number $R_{v}$, and the major outbreak size $\tau_{v}$, are determined. From this it is possible to determine the (strategy-specific) critical vaccination coverage $v_{\mathrm{c}}$, which determines the necessary proportion to vaccinate in order to surely prevent a major outbreak, so $v_{\mathrm{c}}=\inf _{v}\left\{v ; R_{v} \leq 1\right\}$.

Stochastic epidemic models on networks with prespecified degree distributions have mainly been studied in the physics literature (e.g. [7], [17], and [19]), with [1] being one exception. Some of the problems studied in the present paper have been analysed before whereas others have not, in particular the final size proportion $\tau_{v}$ as a function of $v$. Besides contributing some new results, in this paper we also aim to give formal proofs of results which have previously only been obtained heuristically.

The rest of the paper is structured as follows. In Section 2 we define the models for the random graph, the epidemic, and the vaccination strategies. In Section 3 we present the main results and some heuristic motivations, and give some examples and illustrations. The proofs are given in Sections 4 and 5.

\section{Models}

\subsection{Graphs}

Let $G$ denote a random multigraph, allowing for multiple edges and loops, and let $n=|G|$ denote the number of vertices of $G$, i.e. the population size. Later we shall consider limits as $n$ tends to $\infty$. We define our random multigraph as follows. Let $n \in \mathbb{N}$, and let $\left(d_{i}\right)_{1}^{n}=\left(d_{i}^{(n)}\right)_{1}^{n}$ be a sequence of nonnegative integers such that $\sum_{i=1}^{n} d_{i}$ is even. We define a random multigraph with given degree sequence $\left(d_{i}\right)_{1}^{n}$, denoted by $G^{*}\left(n,\left(d_{i}\right)_{1}^{n}\right)$, by the configuration model (see, e.g. [18]): take a set of $d_{i}$ half edges for each vertex $i$, and combine the half edges into pairs by a uniformly random matching of the set of all half edges. 
Note that $G^{*}\left(n,\left(d_{i}\right)_{1}^{n}\right)$ does not exactly have the uniform distribution over all multigraphs with the given degree sequence; there is a weight with a factor $1 / j$ ! for every edge of multiplicity $j$, and a factor $\frac{1}{2}$ for every loop (see [12, Section 1]). However, conditioned on the multigraph being a (simple) graph, we obtain a uniformly distributed random graph with the given degree sequence, which we denote by $G\left(n,\left(d_{i}\right)_{1}^{n}\right)$. It is also worth mentioning that the distribution of $G^{*}\left(n,\left(d_{i}\right)_{1}^{n}\right)$ is the same as the one obtained by sampling the edges as ordered pairs of vertices uniformly with replacement, and then conditioning on the vertex degrees being correct.

Let us write $2 m:=\sum_{i=1}^{n} d_{i}$, so that $m=m(n)$ is the number of edges in the multigraph $G^{*}\left(n,\left(d_{i}\right)_{1}^{n}\right)$. We assume that we are given $\left(d_{i}\right)_{1}^{n}$, satisfying the following regularity conditions; cf. [15] and [16].

Condition 2.1. For each $n,\left(d_{i}\right)_{1}^{n}=\left(d_{i}^{(n)}\right)_{1}^{n}$ is a sequence of nonnegative integers such that $\sum_{i=1}^{n} d_{i}$ is even and, for some probability distribution $\left(p_{j}\right)_{j=0}^{\infty}$ independent of $n$, and with $n_{j}:=\#\left\{i: d_{i}=j\right\}$,

(i) $n_{j} / n \rightarrow p_{j}$ for every $j \geq 0$ as $n \rightarrow \infty$,

(ii) $\mu:=\sum_{j} j p_{j} \in(0, \infty)$,

(iii) $2 m / n \rightarrow \mu$ as $n \rightarrow \infty$,

(iv) $p_{2}<1$.

Remark 2.1. Note that $2 m=\sum_{i} d_{i}=\sum_{j} j n_{j}$. Thus, Condition 2.1 implies that the sum $\sum_{j} j n_{j} / n$ converges uniformly for $n \geq 1$, i.e.

$$
\lim _{J \rightarrow \infty} \sup _{n} \sum_{j>J} \frac{j n_{j}}{n}=0 .
$$

Conversely, (2.1) together with Condition 2.1(i) and Condition 2.1(ii) implies Condition 2.1(iii). (This follows from, e.g. [8, Theorem 5.5.4], taking $X_{n}$ to be the degree of a random vertex.)

Note that our condition is slightly weaker than the one in [15] and [16]; they also assume (in an equivalent formulation) that if $\sum_{j} j^{2} p_{j}<\infty$ then the sums $\sum_{j} j^{2} n_{j} / n$ converge uniformly; moreover, they assume that $j^{2} n_{j} / n \rightarrow j^{2} p_{j}$ uniformly.

Condition 2.1 is all we need to study the random multigraph $G^{*}\left(n,\left(d_{i}\right)_{1}^{n}\right)$. In order to treat the random simple graph $G\left(n,\left(d_{i}\right)_{1}^{n}\right)$, which is our main model, we need an additional assumption.

Condition 2.2. $\sum_{i} d_{i}^{2}=O(n)$.

Note that $\sum_{i} d_{i}^{2}=\sum_{j} j^{2} n_{j}$, so Conditions 2.1 and 2.2 imply, by Fatou's lemma, that $\sum_{j} j^{2} p_{j}<\infty$; in other words, the asymptotic degree distribution has finite variance.

When Conditions 2.1 and 2.2 hold, the probability that $G^{*}\left(n,\left(d_{i}\right)_{1}^{n}\right)$ is a simple graph (i.e. without loops or multiple edges) is bounded away from 0 (see Section 5.2 for details) and, thus, all results that can be stated in terms of convergence in probability for $G^{*}\left(n,\left(d_{i}\right)_{1}^{n}\right)$ transfer to the random simple graph $G\left(n,\left(d_{i}\right)_{1}^{n}\right)$ too.

\subsection{Alternative graph models}

In the remainder of the paper we will consider $G\left(n,\left(d_{i}\right)_{1}^{n}\right)$ as our underlying graph model, but we believe that similar results also hold for other random graph models and that they could be 
proved by suitable modifications of the branching process arguments below. Good candidates are the classical random graphs, $G(n, p)$ and $G(n, m)$, with $p=\mu / n$ and $m=n \mu / 2$ (rounded to an integer), and random graphs of the general type $G(n, \kappa)$ defined in [5]. We will not pursue this here, and leave such attempts at modifying the proofs to the interested reader, but we will discuss one interesting case (including $G(n, p)$ ), where the result easily follows from the results proved below for $G\left(n,\left(d_{i}\right)_{1}^{n}\right)$.

This example is a random graph defined in [6, Section 3], see also [5, Subsection 16.4], as follows. Let $W$ be a nonnegative random variable with finite expectation $\mu_{W}:=\mathrm{E} W$. Firstly, we assign random weights $W_{i}, i=1, \ldots, n$, to the vertices; these weights are independent and identically distributed with the same distribution as $W$. Secondly, given $\left\{W_{i}\right\}_{1}^{n}$, we draw an edge between vertices $i$ and $j$ with probability

$$
p_{i j}:=\frac{W_{i} W_{j}}{n+W_{i} W_{j}} ;
$$

this is done independently (conditioned on $\left\{W_{i}\right\}$ ) for all pairs $\{i, j\}$ with $1 \leq i<j \leq n$. We denote this random graph by $G_{W}(n)$. It is easily seen that [6] (2.2) implies that all graphs with a given degree sequence $\left(d_{i}\right)_{1}^{n}$ have the same probability; in fact, if $G$ is any graph with degree sequence $\left(d_{i}\right)_{1}^{n}$ then

$$
\mathrm{P}\left(G_{W}(n)=G \mid\left(W_{i}\right)_{i=1}^{n}\right)=\frac{n^{\left(\begin{array}{c}
n \\
2
\end{array}\right)-(1 / 2) \sum_{i} d_{i}} \prod_{i} W_{i}^{d_{i}}}{\prod_{i<j}\left(n+W_{i} W_{j}\right)} .
$$

Hence, if we denote the (random) vertex degrees by $D_{1}, \ldots, D_{n}$ then, conditioned on $D_{i}=$ $d_{i}, i=1, \ldots, n$, we have a random graph $G\left(n,\left(d_{i}\right)_{1}^{n}\right)$. Moreover, it is not difficult to verify that Condition 2.1 holds in probability, with $\left(p_{j}\right)_{0}^{\infty}$ the $\operatorname{Po}\left(\mu_{W} W\right)$ mixed Poisson distribution and $\mu=\mu_{W}^{2}$; see [5, Theorem 3.13] and [6, Theorem 3.1]. In other words, $n_{j} / n \stackrel{\mathrm{P}}{\rightarrow} p_{j}$ and $2 m / n=n^{-1} \sum_{i} d_{i} \stackrel{\mathrm{P}}{\rightarrow} \mu$, where $\stackrel{\text { P }}{\rightarrow}$ ' denotes convergence in probability. From now on assume that $\mathrm{E} W^{2}<\infty$; it may then be shown, by similar arguments, that $n^{-1} \sum_{i} d_{i}^{2} \stackrel{\mathrm{P}}{\rightarrow} \mu_{w}^{2}\left(\mathrm{E} W^{2}+1\right)$. Using the Skorohod coupling theorem (see, e.g. [13, Theorem 4.30]), we can assume that these limits hold almost surely (a.s.); hence, Conditions 2.1 and 2.2 hold a.s. Consequently, by conditioning on $\left(D_{1}, \ldots, D_{n}\right)$, we can apply the results proved in the present paper for $G\left(n,\left(d_{i}\right)_{1}^{n}\right)$, and it follows that the theorems, below, hold for the random graph $G_{W}(n)$ too, with $\left(p_{j}\right)$ and $\mu$ as given above.

Furthermore, it is easy to see that this remains true if (2.2) is modified to

$$
p_{i j}:=\min \left(\frac{W_{i} W_{j}}{n}, 1\right)
$$

we may use suitable couplings and compare the random graph defined by (2.3) with the ones defined by (2.2) for the same $W_{i}$ (giving a lower bound), or by (2.2) with $W_{i}$ replaced by $(1+\varepsilon) W_{i}$ (giving an upper bound, assuming, as we may, that $W_{i} \leq \sqrt{\varepsilon n}$ ), and then letting $\varepsilon \searrow 0$; we omit the details. More precisely, it can be shown that [10] under the assumptions given above on $\left(W_{i}\right)$, the random graphs defined by (2.2) and (2.3) are asymptotically equivalent in a strong sense (i.e. the total variation distance tends to 0). Random graphs defined by (2.3) and minor variations of it have been studied by several authors; see [5, Subsection 16.4] and the references therein. Note that the special (deterministic) case in which $W=\sqrt{\mu}$ for a constant $\mu>0$ gives the classical random graph $G(n, \mu / n)$. The results in this paper thus hold for $G(n, \mu / n)$ too, with $\left(p_{j}\right)$ a $\operatorname{Po}(\mu)$ distribution; in other words, with $D$ defined as in Section 3 , $D \sim \operatorname{Po}(\mu)$. 


\subsection{Epidemic model}

We consider an infectious disease that spreads along the edges of a graph $G$. In this paper we will assume that $G=G\left(n,\left(d_{i}\right)_{1}^{n}\right)$ is the random graph defined above, where we condition the graph $G^{*}\left(n,\left(d_{i}\right)_{1}^{n}\right)$ on being simple. The vertices of $G$ represent the individuals in the population, and the edges represent friendships through which the infection might spread.

The disease has its course in the following way. Initially, one randomly chosen individual (vertex) is infected from the outside. This individual then spreads the disease to each of his or her friends independently and with the same probability $p$. Those who become infected make up the first generation infected in the epidemic. These individuals then do the same thing to their not yet infected friends thus infecting a second generation, and so forth. Note that an individual can only become infected once-we then consider such an individual either recovered and immune (or dead). This epidemic continues until there are no new infections in a generation, when it stops. Since the population is finite, this occurs after a finite number of generations (less than or equal to $n$, where $n=|G|$ is the size of the population). The individuals who become infected during the course of the epidemic make up the total outbreak, and the number of such individuals is called the final size of the epidemic.

Note that each edge is a possible path of infection at most once, namely, when the first of its endpoints has been infected. Hence, for every edge in $G$, we may just as well determine in advance whether it will spread the disease or not, provided that one of the endpoints becomes infected. Equivalently, we may consider the graph $G_{p}$ obtained by randomly deleting edges from $G$, with each edge kept with probability $p$, independently of the others. The final size of the epidemic is thus the size of the component of $G_{p}$ containing the initially infected individual.

\subsection{Vaccination strategies}

Now assume that a perfect vaccine is available. By this we mean that an individual who is vaccinated is completely protected from (i.e. immune to) the disease and is not able to spread the disease further. We assume that a part of the population is vaccinated before the epidemic starts, or as soon as the first individual is infected. The epidemic progresses as defined above, with the only difference that infected individuals can only infect unvaccinated friends.

Note that for the study of the epidemic in the vaccinated population, we may simply remove all vaccinated individuals from $G$ (and edges connected to these individuals). If we let $G_{\mathrm{V}}$ denote the remaining graph, and we assume that the initially infected individual $x$ is not vaccinated, the final size of the epidemic is thus the size of the component of $G_{\mathrm{V} ; p}:=\left(G_{\mathrm{V}}\right)_{p}$ that contains $x$. Thus, we have to study the combined effect on $G$ of vertex deletion by the vaccination and edge deletion by the randomness of infection.

The goal is to contain the disease, so that the final size of the epidemic is small, and it is preferable to do this with a rather small number of vaccinations. For this we look at different local vaccination strategies. The first two strategies are local in the sense that they require no global knowledge of the social network $G$ (which is rarely available in applications, [18, Section 8.2]), and the latter two strategies select friendships rather than individuals at random, which may also be thought of as needing only local information. We let $V$ denote the (usually random) number of vaccinations.

2.4.1. Uniform vaccination. Let us assume that we sample a fraction $c \in[0,1]$ chosen uniformly in the population without replacement and that this fraction is immunised, so that the fraction $v$ being immunised satisfies $v=c$. This vaccination strategy is the most commonly studied vaccination strategy owing to its simplicity [18, Section 8.2]. 
More precisely, for convenience, we assume that each individual is vaccinated with a given probability $v$, independently of each other. The number of vaccinations $V$ is thus $\operatorname{Bi}(n, v)$, and $V / n \stackrel{\mathrm{P}}{\rightarrow} v$ as $n \rightarrow \infty$ (with $v$ fixed). We denote the remaining graph of unvaccinated individuals by $G_{v}^{\mathrm{U}}$; this is thus obtained from $G$ by random vertex deletions. Remember that our main concern is with the graph $G_{v ; p}^{\mathrm{U}}=\left(G_{v}^{\mathrm{U}}\right)_{p}$; this is obtained from $G$ by random vertex and edge deletions, independently for all vertices and edges. (In this case it does not matter whether we delete edges or vertices first.)

2.4.2. Acquaintance vaccination. It is intuitively clear that a better vaccination strategy would be to vaccinate the individuals with the highest degrees (i.e. with the most friends) since this would reduce potential spread the most. However, for this targeted vaccination strategy to be achievable the whole social graph (or at least the degrees of all individuals) would have to be known, and this is rarely the case [18, Section 8.2]. A different strategy aiming at vaccinating individuals with high degree, but still only using local graph-knowledge from selected individuals, proposed by Cohen et al. [7], goes under the name of acquaintance vaccination. In this vaccination strategy a fraction $c$ of individuals are sampled and, for each sampled individual, one of his or her friends, chosen randomly among all friends, is vaccinated. Of course it may happen that some individuals are chosen more than once for immunisation (being selected as friends of more than one individual) so the fraction $v=v(c)$ actually immunised is smaller than $c$. This vaccination strategy has two slightly different variants depending on whether the 'fraction' $c$ is chosen with or without replacement. We will use the version with replacement. For this case, the 'fraction' $c$ may in fact exceed 1 without having everyone vaccinated (individuals who are selected more than once are asked for friends independently each time and the friends not yet immunised are vaccinated). To be precise, we let the number of individuals sampled be Poisson distributed $\operatorname{Po}(c n)$, with $c \in[0, \infty)$. Equivalently, each individual is sampled $\mathrm{Po}(c)$ times, and each time reports a randomly chosen friend. Again, for simplicity, we assume that each individual does this with replacement. Consequently, an individual with degree $d$ will report each of his or her friends $\operatorname{Po}(c / d)$ times, and these random numbers are all independent. (An individual that is sampled but has no friends is ignored. An individual is only vaccinated once, even if he or she is reported several times.)

For any initial graph $G$ and $0 \leq c<\infty$, we denote the remaining graph of unvaccinated individuals by $G_{c}^{\mathrm{A}}$. Furthermore, we write $G_{c ; p}^{\mathrm{A}}=\left(G_{c}^{\mathrm{A}}\right)_{p}$ for the graph obtained by additional edge deletions. (For acquaintance vaccination, the order of the deletions is important, since the vaccination strategy uses all edges, without knowing whether they may be selected to transmit the disease or not.)

2.4.3. Edgewise vaccination. In some situations it may be possible to observe, or at least sample, the edges representing friendships. If this is the case, another reasonable vaccination strategy is to sample a number of the edges and then either vaccinate both endpoints or one (randomly selected) endpoint; we denote these two versions by E1 and E2, respectively.

For E2, we assume that we sample each edge with probability $1-\alpha$, where $\alpha \in(0,1]$ is a fixed number. (Equivalently, we sample $\operatorname{Po}(\mathrm{cm})$ edges with replacement, with $\alpha=\mathrm{e}^{-c}$.) For $\mathrm{E} 1$, we assume, for simplicity, that we sample $\operatorname{Po}(2 \mathrm{~cm})$ edges with replacement; thus, each end of each edge is sampled with probability $1-\alpha=1-\mathrm{e}^{-c}$, independently of all other edge ends. Hence, for both versions, a vertex with degree $d$ is unvaccinated with probability $\alpha^{d}$, and, for $E 1$, this is independent of all other vertices. 
For an initial graph $G$ and $0<\alpha \leq 1$, we denote the remaining graph of unvaccinated individuals by $G_{\alpha}^{\mathrm{E} 1}$ and $G_{\alpha}^{\mathrm{E} 2}$, for the two versions. Furthermore, we write, for $j=1,2$, $G_{\alpha ; p}^{\mathrm{E} j}=\left(G_{\alpha}^{\mathrm{Ej}}\right)_{p}$ for the graph obtained by additional edge deletions.

\section{Main results}

We now state our main results together with heuristic motivations. We assume that the underlying graph is the random graph $G\left(n,\left(d_{i}\right)_{1}^{n}\right)$ and that Conditions 2.1 and 2.2 hold. Complete proofs are given in Section 5.

\subsection{Original epidemic model}

Assume that $n$, the number of nodes, is large. The regularity assumption on the degrees of the graph (Condition 2.1) implies that no separate node will contain a large fraction of all edges; see (2.1). This in turn implies that self loops, multiple edges, and short cycles will be rare.

The epidemic starts by a randomly selected individual being infected from outside, so this individual has (approximately) the degree distribution $\left(p_{j}\right)_{j=0}^{\infty}$. The friends of this individual, or friends of any given individual, have the size biased degree distribution $\left(\tilde{p}_{j}\right)_{j=0}^{\infty}$, where

$$
\tilde{p}_{j}=\frac{j p_{j}}{\sum_{k} k p_{k}}
$$

Let $D$ and $\tilde{D}$ be random variables having degree distributions $\left(p_{j}\right)_{j=0}^{\infty}$ and $\left(\tilde{p}_{j}\right)_{j=0}^{\infty}$, respectively. Thus (asymptotically), we can interpret $D$ as the number of friends of a random person, while we can interpret $\tilde{D}$ as the number of friends of a random friend of a given person. Then, given that $D=d$, the number of individuals that the initially infected individual infects is $\operatorname{Bi}(d, p)$, and the unconditional distribution is hence mixed binomial $\operatorname{MixBi}(D, p)$. Those then infected, as well as the infected in the following generations, have degree distribution $\left(\tilde{p}_{j}\right)_{j=0}^{\infty}$. Given that $\tilde{D}=\tilde{d}$, the number of individuals an infected individual infects in the next generation has distribution $\operatorname{Bi}(\tilde{d}-1, p)$. This follows because the infected individual was infected by one of his or her friends (which cannot become reinfected) and, since short cycles are rare, it is very unlikely that any of the remaining $\tilde{d}-1$ friends have already been infected. Unconditionally, the number infected in the next generation is hence $\operatorname{MixBi}(\tilde{D}-1, p)$. Furthermore, the property that short cycles are unlikely implies that the number of infections caused by different individuals are (approximately) independent random variables.

The above paragraph motivates why the early stages of the epidemic may be approximated by a branching process (e.g. [3]), as is common for epidemic models (e.g. [2, Chapter 3]), where 'giving birth' corresponds to infecting someone. The branching process is a simple GaltonWatson process starting with one ancestor having offspring distribution $X \sim \operatorname{MixBi}(D, p)$ and the following generations having offspring distribution $\tilde{X} \sim \operatorname{MixBi}(\tilde{D}-1, p)$. The mean of this latter offspring distribution plays an important role in branching process theory and also in epidemic theory where it is denoted by $R_{0}$ and called the basic reproduction number. Using (3.1), we obtain

$$
R_{0}=\mathrm{E}(\tilde{X})=p \mathrm{E}(\tilde{D}-1)=p\left(\frac{\sum_{j} j^{2} p_{j}}{\mu}-1\right)=p\left(\mu+\frac{\operatorname{var}(D)-\mu}{\mu}\right),
$$

where $\mu=\mathrm{E}(D)=\sum_{k} k p_{k}$ and $\operatorname{var}(D)=\sum_{j} j^{2} p_{j}-\mu^{2}$ (a closely related expression is obtained in [1]). The branching process is subcritical, critical, or supercritical depending on whether $R_{0}<1, R_{0}=1$, or $R_{0}>1$, respectively. For the epidemic, this means that a 
major outbreak infecting a nonnegligible fraction of the community is possible if and only if $R_{0}>1$. Note that, for fixed $\mu, R_{0}$ is increasing in $\operatorname{var}(D)$, so the more variance in the degree distribution, the higher $R_{0}$, and if the degree distribution has infinite variance then $R_{0}=\infty$ (a case not treated in the present manuscript owing to Condition 2.2).

The probability $\pi$ that the branching process dies out is derived in the standard way as follows. First we derive the probability $\tilde{\pi}$ that a branching process with all individuals having offspring distribution $\tilde{X}$ dies out. This is obtained by conditioning on the number of individuals born in the first generation: for the branching process to die out, all branching processes initiated by the individuals of the first generation must die out, i.e.

$$
\tilde{\pi}=\sum_{k=0}^{\infty} \tilde{\pi}^{k} \mathrm{P}(\tilde{X}=k) \text {. }
$$

Let $f_{\tilde{X}}(\cdot)$ denote the probability generating function for $\tilde{X}$, and let $f_{D}(\cdot)$ denote the probability generating function of the original degree distribution $D$. Then we see that $\tilde{\pi}$ is a solution to the equation $f_{\tilde{X}}(t)=t$, and it is known, from branching process theory (e.g. [3, Theorem I.5.1]), that it is the smallest nonnegative such solution. The fact that $\tilde{X}$ is $\operatorname{Mix} \operatorname{Bi}(\tilde{D}-1, p)$ implies that

$$
f_{\tilde{X}}(t)=\mathrm{E}\left(t^{\tilde{X}}\right)=\mathrm{E}\left(\mathrm{E}\left(t^{\tilde{X}} \mid \tilde{D}\right)\right)=\mathrm{E}\left((p t+1-p)^{\tilde{D}-1}\right)=\mathrm{E}\left((1-p(1-t))^{\tilde{D}-1}\right) .
$$

Furthermore, for $0<a<1$,

$$
\mathrm{E}\left(a^{\tilde{D}-1}\right)=\sum_{k} a^{k-1} \frac{k p_{k}}{\mu}=\frac{\mathrm{d}}{\mathrm{d} a} \sum_{k} a^{k} \frac{p_{k}}{\mu}=\frac{\mathrm{d}}{\mathrm{d} a} \frac{f_{D}(a)}{\mu}=\frac{f_{D}^{\prime}(a)}{\mu}=\frac{f_{D}^{\prime}(a)}{f_{D}^{\prime}(1)} .
$$

In terms of $f_{D}(\cdot)$ the probability $\tilde{\pi}$ that the branching process dies out is hence the smallest nonnegative solution to

$$
\frac{f_{D}^{\prime}(1-p(1-\tilde{\pi}))}{f_{D}^{\prime}(1)}=\tilde{\pi} .
$$

The probability $\pi$ that the branching process, in which the ancestor has different offspring distribution $X$, dies out is obtained from $\tilde{\pi}$ by conditioning on the number of offspring of the ancestor:

$$
\begin{aligned}
\pi & =\sum_{k} \tilde{\pi}^{k} \mathrm{P}(X=k) \\
& =\mathrm{E}\left(\tilde{\pi}^{X}\right) \\
& =\mathrm{E}\left(\mathrm{E}\left(\tilde{\pi}^{X} \mid D\right)\right) \\
& =\mathrm{E}\left((p \tilde{\pi}+1-p)^{D}\right) \\
& =f_{D}(1-p(1-\tilde{\pi})) .
\end{aligned}
$$

We now look at the final size of the epidemic in case it takes off, corresponding to the case that the branching process grows beyond all limits. We do this by considering the epidemic from a graph representation. The social structure was represented by a random graph $G$. If this graph is thinned by removing each edge independently with probability $1-p$, we obtain a thinned graph denoted by $G_{p}$. Edges in $G_{p}$ represent the potential spread of infection: if one of the nodes becomes infected from elsewhere, its neighbour will become infected. As a consequence, the final outbreak of the epidemic will consist of all nodes in $G_{p}$ that are connected 
to the initially infected. From random graph theory, it is known that if $R_{0}>1$, there will be exactly one connected component of order $n$, the giant component, and all remaining connected components will be of smaller order. If $R_{0} \leq 1$, there will be no giant component. The initially infected individual was chosen uniformly in the community, so he or she will belong to the giant component with a probability that equals the relative size of the giant component. Conversely, the initially infected individual belongs to the giant component if and only if its branching process of new infections grows beyond all limits, and from before we know that this happens with probability $1-\pi$, where $\pi$ is as defined in (3.4). From this it follows that the asymptotic final proportion infected, $\tau$, equals $1-\pi$. So, $\tau$ is both the probability of a major outbreak and the relative size of the outbreak in case a major outbreak occurs.

The above arguments motivate the following theorem, which we prove in Section 5, and where $Z_{n}$ denotes the final number infected in the epidemic.

Theorem 3.1. If $R_{0} \leq 1$ then $Z_{n} / n \stackrel{\mathrm{P}}{\rightarrow} 0$. If $R_{0}>1$ then $Z_{n} / n$ converges to a two-point distribution $Z$ for which

$$
\mathrm{P}(Z=0)=\pi \quad \text { and } \quad \mathrm{P}(Z=\tau)=\tau,
$$

where $\pi$ is defined by (3.3) and (3.4), and $\tau=1-\pi$.

\subsection{Uniform vaccination}

Prior to the arrival of the infectious disease, each individual is vaccinated independently and with the same probability $v$, which implies that the total number vaccinated $V$ is $\operatorname{Bi}(n, v)$ and, from the law of large numbers, the random proportion vaccinated $V / n \stackrel{\mathrm{P}}{\rightarrow} v$.

Vaccinated individuals, and the edges connecting them, can be removed from the graph since there will be no spreading between these individuals and their friends in either direction. As a consequence, an individual who originally had $d$ friends now has $\operatorname{Bi}(d, 1-v)$ unvaccinated friends. If an individual becomes infected during the early stages of the epidemic, he or she will infect each of his or her unvaccinated friends independently with probability $p$. Given that the initially infected individual has degree $d$, he or she will hence infect $\operatorname{Bi}(d, p(1-v))$ friends, so without the conditioning he or she will infect a mixed binomial number $X_{v} \sim$ $\operatorname{Mix} \operatorname{Bi}(D, p(1-v))$. Similarly, during the early stages, an infected individual with degree $d$ will infect $\operatorname{Bi}(d-1, p(1-v))$, and unconditionally an individual has degree distribution $\left\{\tilde{p}_{k}\right\}$, so the unconditional number he or she will infect, $\tilde{X}_{v}$, will be $\operatorname{Mix} \operatorname{Bi}(\tilde{D}-1, p(1-v))$.

It is seen that we have the same type of distributions as in the case without vaccination. As a consequence, all results for the case with uniform vaccination can be obtained from the case without vaccination simply by replacing $p$ by $p(1-v)$. Hence, we find that the reproduction number $R_{v ; p}^{\mathrm{U}}$ after vaccinating a fraction $v$ chosen uniformly satisfies

$$
R_{v ; p}^{\mathrm{U}}=\mathrm{E}\left(\tilde{X}_{v}\right)=(1-v) R_{0}=p(1-v)\left(\mu+\frac{\operatorname{var}(D)-\mu}{\mu}\right) .
$$

The probability $\tilde{\pi}_{v ; p}^{\mathrm{U}}$ that the epidemic never takes off, assuming that the initially infected individual has $\tilde{X}_{v}$ unvaccinated friends, is the smallest solution to

$$
\frac{f_{D}^{\prime}\left(1-p(1-v)\left(1-\tilde{\pi}_{v ; p}^{\cup}\right)\right)}{f_{D}^{\prime}(1)}=\tilde{\pi}_{v ; p}^{\cup} .
$$


The probability $\pi_{v ; p}^{U}$ that the epidemic never takes off, if the initially infected individual is selected randomly among the unvaccinated, is given by

$$
\pi_{v ; p}^{\cup}=f_{D}\left(1-p(1-v)\left(1-\tilde{\pi}_{v ; p}^{U}\right)\right)
$$

where $\tilde{\pi}_{v ; p}^{\cup}$ is the smallest solution to (3.5). Finally, the final size is determined from the probability of a major outbreak, as before. This means that the final proportion infected (among the unvaccinated!) will converge to $1-\pi_{v ; p}^{\mathrm{U}}$ in case of a major outbreak. We have the following corollary, where $Z_{n}^{\mathrm{U}}(v)$ denotes the final number infected in the epidemic, where each individual was vaccinated independently with probability $v(0 \leq v<1)$ prior to the outbreak, and where the initially infected individual was chosen randomly among the unvaccinated.

Theorem 3.2. If $R_{v ; p}^{\cup} \leq 1$ then $Z_{n}^{\cup}(v) /((1-v) n) \stackrel{\mathrm{P}}{\rightarrow} 0$. If $R_{v ; p}^{\cup}>1$ then $Z_{n}^{\mathrm{U}}(v) /((1-v) n)$ converges to a two-point distribution $Z_{v ; p}^{\cup}$ for which

$$
\mathrm{P}\left(Z_{v ; p}^{\mathrm{U}}=0\right)=\pi_{v ; p}^{\mathrm{U}} \text { and } \mathrm{P}\left(Z_{v ; p}^{\mathrm{U}}=\tau_{v ; p}^{\mathrm{U}}\right)=\tau_{v ; p}^{\mathrm{U}} \text {, }
$$

where $\pi_{v ; p}^{\mathrm{U}}$ is defined by (3.5) and (3.6), and $\tau_{v ; p}^{\mathrm{U}}=1-\pi_{v ; p}^{\mathrm{U}}$.

\subsection{Acquaintance vaccination}

Recall that each individual is sampled, independently, $\operatorname{Po}(c)$ number of times, where $0 \leq$ $c<\infty$, so in total $\operatorname{Po}(n c)$ individuals are sampled. Each time an individual is sampled, a randomly chosen friend of the individual is selected and vaccinated (unless they have already been vaccinated). The effect of this strategy is that vaccinated individuals, being selected as somebody's friend, have the size biased degree distribution $\left(\tilde{p}_{j}\right)_{j=0}^{\infty}$, where $\tilde{p}_{j}=j p_{j} / \sum_{k} k p_{k}$, rather than the original degree distribution $\left\{p_{k}\right\}$ for uniformly selected individuals. The proportion vaccinated, $v=v(c)$, is obtained as follows. An individual avoids being vaccinated if he or she is not vaccinated 'through' any of his or her friends. The friends of the individual have independent degree distributions $\left(\tilde{p}_{j}\right)_{j=0}^{\infty}$, and the probability of not being vaccinated 'through' an individual with degree $k$ is $\mathrm{e}^{-c / k}$ (the number of vaccination attempts on a specific friend is $\operatorname{Po}(c / k))$. It follows that the probability of avoiding being vaccinated from one friend equals

$$
\alpha=\alpha(c)=\sum_{k=1}^{\infty} \mathrm{e}^{-c / k} \tilde{p}_{k}=\sum_{k=1}^{\infty} \mathrm{e}^{-c / k} \frac{k p_{k}}{\mu} .
$$

(Note that $\alpha$ has the same interpretation as for $\alpha$ introduced for the edgewise strategies, but it is a different function of $c$.) If the individual in question has $j$ friends, it hence avoids being vaccinated with probability $\alpha^{j}$. The proportion $1-v(c)$ not being vaccinated equals the probability that a randomly selected individual is not vaccinated, which hence equals

$$
1-v(c)=\sum_{j=0}^{\infty} \alpha^{j} p_{j}=f_{D}(\alpha),
$$

where, as before, $f_{D}(\cdot)$ is the probability generating function of a random variable $D$ having distribution $\left(p_{j}\right)_{j=0}^{\infty}$.

Note that in this model, given the graph, individuals are vaccinated independently of each other (although with different probabilities). It easily follows that the actual (random) number $V$ of vaccinated individuals satisfies

$$
\frac{V}{n} \stackrel{\mathrm{P}}{\rightarrow} v(c) \quad \text { as } n \rightarrow \infty
$$


Hence, we will ignore the randomness in $V$ and regard $v(c)$, given by (3.8), as the proportion of vaccinated people.

We now approximate the initial stages of an epidemic, occurring in a community having been vaccinated according to the acquaintance strategy, with a suitable branching process. It is more difficult to find 'the right' branching process approximation for the acquaintance strategy because the vaccination status of an individual depends on the degrees of his or her friends. Therefore, we introduce some convenient terminology.

We say that transmission may take place through an edge, and through its two half edges, if it is one of the edges in $G_{p}$, i.e. one of the randomly selected edges which will spread the disease if one of its endpoints is infected. (Recall that we may assume that this random selection takes place before the start of the infection.) Furthermore, there is a natural correspondence between half edges and directed edges, with a half edge corresponding to the edge it is part of, directed so that it begins with this half edge. We say that a directed edge, or the corresponding half edge, is used for vaccination, if the person at the start of the edge is selected and names the person at the end of the edge, who thus gets vaccinated.

It turns out that a suitable 'individual' in the branching process is a pair $(x, \epsilon)$ consisting of an unvaccinated person $x$ together with a directed edge $\epsilon$ from this person satisfying the conditions that transmission may take place through the edge $\epsilon$ and that $\epsilon$ is not used for vaccination. It is worth noting that a person may be part of several 'individuals' in the branching process (if the person was not vaccinated and has several friends such that the connecting edges satisfy the conditions above). See Figure 1 for an illustration of an individual (Figure 1(a)) and situations where the individual 'gives birth' to two individuals (Figure 1(b)) and zero individuals (Figure 1(c)). In Figure 1(b) the 'individual' to the left gives birth to two individuals: the person in the middle together with the up-going edge, and the person in the middle together with the edge to the right. These two edges are parts of individuals since the edges are open for transmission and the middle person did not name the friends at the other end for vaccination. The down-going edge is not part of an individual since the person in the middle named the friend below for vaccination. In Figure 1(c) no individual is born since the middle person is vaccinated (being named for vaccination by one of his or her friends, excluding the person to the left).

In order to analyse the corresponding branching process we have to determine the distribution of how many new 'individuals' one 'individual' will infect during the early stages of the epidemic, assuming a large population (i.e. large $n$ ). First we determine the distribution of the degree $K$ of the friend $z$ at the other end of the edge $\epsilon$ of our 'individual' $(x, \epsilon)$. We know that person $x$ of our 'individual' is unvaccinated, so the edge $\epsilon$ has not been used for vaccination backwards, i.e. in the opposite direction. As a consequence, we have to condition on this, and then the friend $z$ at the other end of $\epsilon$ has degree $K=k$ with probability

$$
\mathrm{P}(K=k)=\frac{\tilde{p}_{k} \mathrm{e}^{-c / k}}{\sum_{j=1}^{\infty} \tilde{p}_{j} \mathrm{e}^{-c / j}}=\frac{\tilde{p}_{k} \mathrm{e}^{-c / k}}{\alpha}, \quad k=1,2, \ldots,
$$

i.e. the size biased degree distribution conditional on not having vaccinated backwards. In order for this friend $z$ to create new 'individuals', he or she must not have been vaccinated by any of his or her other $K-1$ friends (by assumption, he or she was not vaccinated from our original individual $x$ ). Conditioned on $K=k$, this occurs with probability $\alpha^{k-1}$. Each of the friend's remaining $k-1$ edges will then be open (i.e. transmission may take place, but it is not used for vaccination) independently, each open with probability $p \mathrm{e}^{-c / k}$. The number of open edges (equal to the number of new 'individuals') is hence $\operatorname{Bi}\left(k-1, p \mathrm{e}^{-c / k}\right)$. If the 


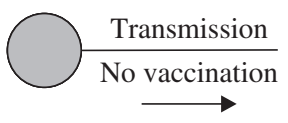

(a)

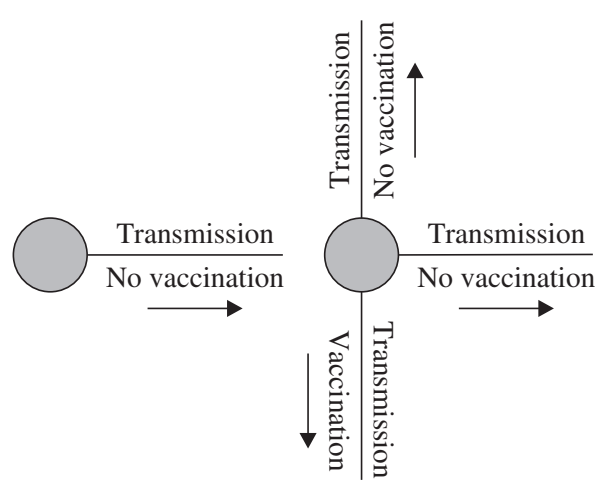

(b)

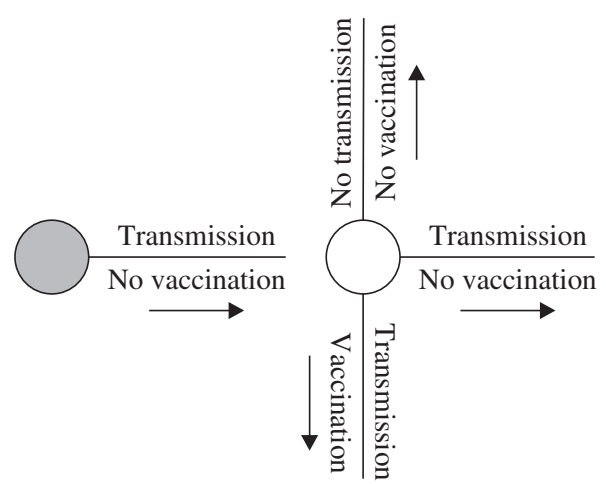

(c)

Unvaccinated individual $\bigcirc$ Vaccinated individual

Figure 1: (a) An illustration of an 'individual' in the branching process. In (b) the left 'individual' has two offspring: the person in the middle with the edge to its right, and the person in the middle with the up-going edge. In (c) no individual is born.

friend is vaccinated (probability $1-\alpha^{k-1}$ ), no new individuals are born. The unconditional number $Y$ of new 'individuals' an individual 'gives birth' to, i.e. the offspring distribution of the approximating branching process, can thus be obtained by conditioning on the $K$ number of friends our friend $z$ has, using (3.10) and recalling that zero individuals are born whenever the friend is vaccinated or if the binomial variable equals 0 :

$$
\begin{gathered}
\mathrm{P}(Y=0)=\sum_{k=1}^{\infty}\left(\left(1-\alpha^{k-1}\right)+\alpha^{k-1}\left(1-p \mathrm{e}^{-c / k}\right)^{k-1}\right) \frac{\tilde{p}_{k} \mathrm{e}^{-c / k}}{\alpha}, \\
\mathrm{P}(Y=j)=\sum_{k=j+1}^{\infty} \alpha^{k-1}\left(\begin{array}{c}
k-1 \\
j
\end{array}\right)\left(p \mathrm{e}^{-c / k}\right)^{j}\left(1-p \mathrm{e}^{-c / k}\right)^{k-1-j} \frac{\tilde{p}_{k} \mathrm{e}^{-c / k}}{\alpha}, \quad j \geq 1 .
\end{gathered}
$$

This offspring distribution determines both $R_{c ; p}^{\mathrm{A}}$, the probability of a major outbreak, and the final size in case of a major outbreak. For instance, the reproduction number is the mean of this distribution, and this mean is obtained by first conditioning on the degree of the node in question. Given that the degree equals $k$, the average number of offspring equals $\alpha^{k-1}(k-1) p \mathrm{e}^{-c / k}$, which gives the following reproduction number:

$$
R_{c ; p}^{\mathrm{A}}=\mathrm{E}(Y)=\sum_{k \geq 1} \alpha^{k-1}(k-1) p \mathrm{e}^{-c / k} \frac{\tilde{p}_{k} \mathrm{e}^{-c / k}}{\alpha}=p \sum_{k \geq 1}(k-1) \alpha^{k-2} \mathrm{e}^{-2 c / k} \tilde{p}_{k}
$$


(see [7]). Let $f_{Y}(a)=\mathrm{E}\left(a^{Y}\right)$ be the probability generating function of this offspring distribution. If the epidemic is started by one 'individual', i.e. one person with one open directed edge, then the probability $\tilde{\pi}_{c ; p}^{\mathrm{A}}$ that the epidemic never takes off is the smallest solution to the equation

$$
\tilde{\pi}_{c ; p}^{\mathrm{A}}=f_{Y}\left(\tilde{\pi}_{c ; p}^{\mathrm{A}}\right) .
$$

If we start with one infected person that is unvaccinated and has degree $j$, then each of its $j$ half edges is open with probability $p \mathrm{e}^{-c / j}$, and the probability that a given half edge does not start a large epidemic is $1-p \mathrm{e}^{-c / j}+p \mathrm{e}^{-c / j} \tilde{\pi}_{c ; p}^{\mathrm{A}}$, so the probability that the epidemic never takes off equals $\left(1-p \mathrm{e}^{-c / j}\left(1-\tilde{\pi}_{c ; p}^{\mathrm{A}}\right)\right)^{j}$ for $j \geq 1$ and equals 1 for $j=0$.

If the initially infected individual is chosen randomly among the unvaccinated as we assume, then the probability that it has degree $j$ is $p_{j} \alpha^{j} / \sum_{j} p_{j} \alpha^{j}$ (see (3.8)) and, thus, the probability that the epidemic never takes off equals

$$
\pi_{c ; p}^{\mathrm{A}}=\frac{p_{0}+\sum_{j \geq 1} p_{j} \alpha^{j}\left(1-p \mathrm{e}^{-c / j}\left(1-\tilde{\pi}_{c ; p}^{\mathrm{A}}\right)\right)^{j}}{\sum_{j} p_{j} \alpha^{j}} .
$$

Finally, using the same reasoning as before, the limiting proportion infected in case of a major outbreak equals $\tau_{c ; p}^{\mathrm{A}}=1-\pi_{c ; p}^{\mathrm{A}}$. We summarise our results in the following theorem, proved in Section 5, where $Z_{n}^{\mathrm{A}}(c)$ denotes the final number infected in the epidemic where vaccination is carried out prior to the outbreak according to the acquaintance vaccination strategy. Recall that $0 \leq c<\infty$ and that $v(c)$, the proportion of the population vaccinated, is given by (3.8), with $\alpha=\alpha(c)$ given by (3.7).

Theorem 3.3. If $R_{c ; p}^{\mathrm{A}} \leq 1$ then $Z_{n}^{\mathrm{A}}(c) /((1-v(c)) n) \stackrel{\mathrm{P}}{\rightarrow} 0$, where $R_{c ; p}^{\mathrm{A}}$ is defined by (3.12). If $R_{c ; p}^{\mathrm{A}}>1$ then $Z_{n}^{\mathrm{A}}(c) /((1-v(c)) n)$ converges to a two-point distribution $Z_{c ; p}^{\mathrm{A}}$ for which $\mathrm{P}\left(Z_{c ; p}^{\mathrm{A}}=0\right)=\pi_{c ; p}^{\mathrm{A}}$ and $\mathrm{P}\left(Z_{c ; p}^{\mathrm{A}}=\tau_{c ; p}^{\mathrm{A}}\right)=\tau_{c ; p}^{\mathrm{A}}$, where $\pi_{c ; p}^{\mathrm{A}}$ is defined by (3.13) and (3.14), and $\tau_{c ; p}^{\mathrm{A}}=1-\pi_{c ; p}^{\mathrm{A}}$.

\subsection{Edgewise vaccination}

Recall that, for both $\mathrm{E} 1$ and $\mathrm{E} 2$, a person with $d$ friends is unvaccinated with probability $\alpha^{d}$ (here $\alpha$ has the same meaning as in the previous subsection, but it can be treated as a free parameter). Thus,

$$
\mathrm{E} V=n \sum_{d} p_{d}\left(1-\alpha^{d}\right)+o(n)
$$

and a simple variance estimate shows that the vaccinated proportion

$$
\frac{V}{n} \stackrel{\mathrm{P}}{\rightarrow} v(\alpha):=\sum_{d} p_{d}\left(1-\alpha^{d}\right)
$$

just as for the acquaintance vaccination strategy; see (3.8) and (3.9).

We define open (directed) edges in the same way as for acquaintance vaccination, and argue as there with the following modifications. The other endpoint of an open edge has just the sizebiased distribution $\left(\tilde{p}_{k}\right)$. If this vertex, $z$ say, has degree $k$, it is unvaccinated with probability $\alpha^{k-1}$, and in this case the number of new open edges originating at $z$ is $\operatorname{Bi}(k-1, p \alpha)$ for $\mathrm{E} 1$ and $\operatorname{Bi}(k-1, p)$ for E2. The difference between the two versions arises because we already know that these edges do not vaccinate $z$, and, for E2, this implies that they do not vaccinate their other endpoint either while, for $\mathrm{E} 1$, this is an independent event having probability $\alpha$. 
We thus have the offspring distributions for E1 and E2 (see (3.11)):

$$
\begin{gathered}
\mathrm{P}\left(Y_{1}=j\right)=\sum_{k=j+1}^{\infty} \tilde{p}_{k} \alpha^{k-1}\left(\begin{array}{c}
k-1 \\
j
\end{array}\right)(p \alpha)^{j}(1-p \alpha)^{k-1-j}, \quad j \geq 1, \\
\mathrm{P}\left(Y_{2}=j\right)=\sum_{k=j+1}^{\infty} \tilde{p}_{k} \alpha^{k-1}\left(\begin{array}{c}
k-1 \\
j
\end{array}\right) p^{j}(1-p)^{k-1-j}, \quad j \geq 1
\end{gathered}
$$

we leave the formulae for $\mathrm{P}\left(Y_{1}=0\right)$ and $\mathrm{P}\left(Y_{2}=0\right)$ to the reader. This gives the reproduction numbers

$$
\begin{aligned}
& R_{\alpha ; p}^{\mathrm{E} 1}=\mathrm{E}\left(Y_{1}\right)=\sum_{k \geq 1} \tilde{p}_{k} \alpha^{k-1}(k-1) p \alpha=p \sum_{k}(k-1) \tilde{p}_{k} \alpha^{k}, \\
& R_{\alpha ; p}^{\mathrm{E} 2}=\mathrm{E}\left(Y_{2}\right)=\sum_{k \geq 1} \tilde{p}_{k} \alpha^{k-1}(k-1) p=p \sum_{k}(k-1) \tilde{p}_{k} \alpha^{k-1} .
\end{aligned}
$$

Note that $R_{\alpha ; p}^{\mathrm{E} 1}=\alpha R_{\alpha ; p}^{\mathrm{E} 2}<R_{\alpha ; p}^{\mathrm{E} 2}$, which shows that, with the same number of vaccinations, $\mathrm{E} 1$ is a better strategy than E2. In particular, the critical vaccination coverage $v_{\mathrm{c}}$ is smaller for $\mathrm{E} 1$ than for E2. An intuitive explanation for why E2 is not as efficient as E1 is that in E2 both individuals of selected friendships are vaccinated, and since an individual is partly protected by friends becoming vaccinated, the second vaccination is less 'efficient'.

We let $\tilde{\pi}_{\alpha ; p}^{\mathrm{E} 1}$ and $\tilde{\pi}_{\alpha ; p}^{\mathrm{E} 2}$ denote the probabilities that the Galton-Watson processes with offspring distributions $Y_{1}$ and $Y_{2}$, respectively, starting with one individual, die out; thus, they are the smallest positive solutions to $t=f_{Y_{1}}(t)$ and $t=f_{Y_{2}}(t)$, where $f_{Y_{1}}$ and $f_{Y_{2}}$ are the corresponding probability generating functions.

If we start with one unvaccinated person $x$ with degree $d$, the number of open edges from $x$ is $\operatorname{Bi}(d, p \alpha)$ for $\mathrm{E} 1$ and $\operatorname{Bi}(d, p)$ for $\mathrm{E} 2$, for the same reason as for the number of new edges above. The probability that the epidemic never takes off is thus $\left(1-p \alpha+p \alpha \tilde{\pi}_{\alpha ; p}^{\mathrm{E} 1}\right)^{d}$ for $\mathrm{E} 1$ and $\left(1-p+p \tilde{\pi}_{\alpha ; p}^{\mathrm{E}}\right)^{d}$ for E2.

If the initially infected individual is chosen randomly among the unvaccinated, we thus find that the probabilities that the epidemic never takes off are

$$
\begin{aligned}
\pi_{\alpha ; p}^{\mathrm{E} 1} & =\frac{\sum_{j} p_{j} \alpha^{j}\left(1-p \alpha\left(1-\tilde{\pi}_{\alpha ; p}^{\mathrm{E} 1}\right)\right)^{j}}{\sum_{j} p_{j} \alpha^{j}}=\frac{f_{D}\left(\alpha\left(1-p \alpha\left(1-\tilde{\pi}_{\alpha ; p}^{\mathrm{E} 1}\right)\right)\right)}{f_{D}(\alpha)}, \\
\pi_{\alpha ; p}^{\mathrm{E} 2} & =\frac{\sum_{j} p_{j} \alpha^{j}\left(1-p\left(1-\tilde{\pi}_{\alpha ; p}^{\mathrm{E} 2}\right)\right)^{j}}{\sum_{j} p_{j} \alpha^{j}}=\frac{f_{D}\left(\alpha\left(1-p\left(1-\tilde{\pi}_{\alpha ; p}^{\mathrm{E} 2}\right)\right)\right)}{f_{D}(\alpha)} .
\end{aligned}
$$

We summarise our results as before, letting $Z_{n}^{\mathrm{E} 1}(\alpha)$ and $Z_{n}^{\mathrm{E} 2}(\alpha)$ denote the final numbers infected in the epidemic for the two strategies. Recall that $v(\alpha)$ is given by (3.15).

Theorem 3.4. For $j=1,2, Z_{n}^{\mathrm{E} j}(\alpha) /((1-v(\alpha)) n) \stackrel{\mathrm{P}}{\rightarrow} 0$ if $R_{\alpha ; p}^{\mathrm{Ej}} \leq 1$, where $R_{\alpha ; p}^{\mathrm{Ej}}$ is defined by (3.16). If $R_{\alpha ; p}^{\mathrm{E}_{j}}>1$ then $Z_{n}^{\mathrm{Ej}}(\alpha) /((1-v(\alpha)) n)$ converges to a two-point distribution $Z_{\alpha ; p}^{\mathrm{E} j}$ for which $\mathrm{P}\left(Z_{\alpha ; p}^{\mathrm{E}_{\mathrm{E} j} j}=0\right)=\pi_{\alpha ; p}^{\mathrm{E}_{j}}$ and $\mathrm{P}\left(Z_{\alpha ; p}^{\mathrm{E}_{j}}=\tau_{\alpha ; p}^{\mathrm{E}_{j}}\right)=\tau_{\alpha ; p}^{\mathrm{E}_{j}}$, where $\pi_{\alpha ; p}^{\mathrm{E}_{j}}$ is defined by (3.17), and $\tau_{\alpha ; p}^{\mathrm{E}_{j}}=1-\pi_{\alpha ; p}$.

\subsection{Examples}

We now compare the performance of the different vaccination strategies using two examples. In the first example we have chosen the degree distribution to be Poisson distributed with mean 


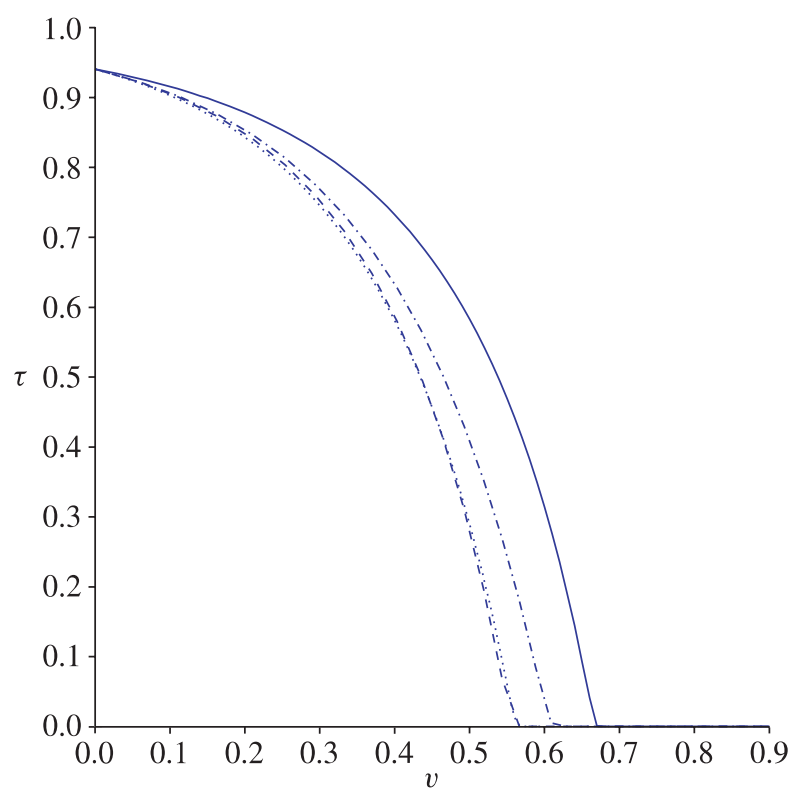

FIGURE 2: Final proportion infected, $\tau$, as a function of the vaccination coverage $v$ for four vaccination strategies: uniform (solid line), acquaintance (dotted line), E1 (dashed line), and E2 (dash-dot line). The degree distribution is $\operatorname{Po}(6)$ and the transmission probability is $p=0.5$.

$\lambda=6$, and the transmission probability to equal $p=0.5$. Using (3.2), we conclude that this implies that $R_{0}=3$. The assumption of Poisson distributed degree means that this applies to the simple $G(n, p=6 / n)$ graph with transmission probability $p=0.5$; in the epidemic literature, this model is known as the Reed-Frost model (e.g. [2, 4-6]). In Figure 2 we show $\tau$, the final proportion infected among the unvaccinated in case of a major outbreak, as a function of the vaccination coverage $v$ for the four different vaccination strategies treated. It is seen that the acquaintance and edgewise E1 strategies perform best in the sense that, for a fixed proportion vaccinated, the proportion $\tau$ becoming infected in case of a major outbreak is smallest for these two strategies. As a consequence, the critical vaccination coverage $v_{\mathrm{c}}=\inf _{v}\left\{v ; R_{v} \leq 1\right\}$ is also smallest for these two strategies. There is no unique ordering of the two strategies- the acquaintance strategy is slightly better for small vaccination coverages and $\mathrm{E} 1$ is slightly better for higher vaccination coverages and hence also has slightly smaller $v_{\mathrm{c}}$. The edgewise strategy E2 is not as good as these two strategies but still better than the uniform vaccination coverage. (Indeed, E2 is always less efficient than E1, see above.) Acquaintance, E1, and E2 all perform better than the uniform strategy, the reason being that they tend to find individuals with high degrees. For the parameter choices of this example, the critical vaccination coverages equal $v_{\mathrm{c}} \approx 0.56$ for the acquaintance and E1 strategies, $v_{\mathrm{c}} \approx 0.61$ for $\mathrm{E} 2$, and $v_{\mathrm{c}} \approx 0.67$ for the uniform vaccination strategy.

In the second example (illustrated in Figure 3) we chose a more heavy-tailed degree distribution having $p_{d} \propto d^{-3.5}$ (in the computations it was truncated at $d=200$ ). The initial values were modified such that $\mathrm{E}(D) \approx 6$, to make it more comparable to the previous example, with a resulting variance equal to 18.9 . The transmission parameter was set at $p=0.5$, as before. Using (3.2), we see that $R_{0} \approx 4.1$. In Figure 3 we see the same type of pattern as in the previous example. However, the difference between the strategies is more pronounced with $v_{\mathrm{c}} \approx 0.50$ 


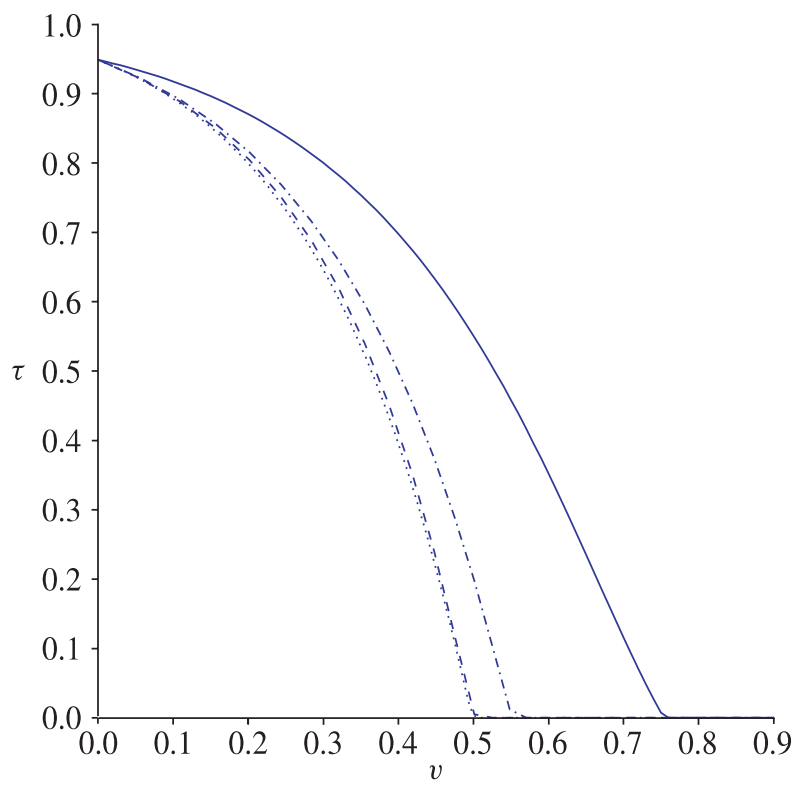

FIGURE 3: Final proportion infected, $\tau$, as a function of the vaccination coverage $v$ for four vaccination strategies: uniform (solid line), acquaintance (dotted line), E1 (dashed line) and E2 (dash-dot line). The degree distribution is heavy tailed $\left(p_{d} \propto d^{-3.5}\right)$ with mean $\mathrm{E}(D) \approx 6$ and $p=0.5$.

for the acquaintance and $\mathrm{E} 1$ strategies, $v_{\mathrm{c}} \approx 0.55$ for $\mathrm{E} 2$, and $v_{\mathrm{c}} \approx 0.75$ for the uniform vaccination strategy. In other words, if the uniform strategy is applied in these two examples, we have to vaccinate more individuals if the degree distribution is heavy tailed, but if one of the other strategies is performed, the heavy-tailed degree distribution requires less vaccinations to surely prevent an outbreak. Another minor difference from the previous example is that, for the present heavy-tailed distribution, the acquaintance strategy is (slightly) better than E1 for all vaccination coverages and, hence, also has a smaller critical vaccination coverage. However, the difference between the two strategies is negligible.

Note that all the $\tau \mathrm{s}$ in both examples denote the proportion of infected individuals among the unvaccinated (in case of an outbreak) and hence can be thought of as an indirect protection from those becoming vaccinated. Of course, by assumption, all vaccinated individuals are also protected from becoming infected.

\section{Preliminaries on branching processes}

As stated above, our method is based on a comparison with branching processes, more precisely Galton-Watson processes; see, e.g. [3, 1-62] for definitions and basic facts. If $\mathfrak{X}$ is a Galton-Watson process started with one initial particle, we let $\mathfrak{X}^{d}$ denote the same branching process with $d$ initial particles, i.e. the union of $d$ independent copies of $\mathfrak{X}$. Furthermore, for any Galton-Watson process $\mathfrak{X}$, we let $|\mathfrak{X}|$ denote its total progeny, i.e. the total number of particles in all generations, and we let $\rho(\mathfrak{X})$ be the survival probability of $\mathfrak{X}$, i.e.

$$
\rho(\mathfrak{X}):=\mathrm{P}(|\mathfrak{X}|=\infty) .
$$


Note that if $\mathfrak{X}$ starts with one particle then

$$
\rho\left(\mathfrak{X}^{d}\right)=1-(1-\rho(\mathfrak{X}))^{d},
$$

since $\mathfrak{X}^{d}$ dies out if and only if all $d$ copies of $\mathfrak{X}$ in it do.

We will need the following simple continuity result, which, although we have failed to find a reference, is presumably well known.

Lemma 4.1. Let $X_{v}$ and $X$ be nonnegative, integer-valued random variables, and let $\mathfrak{X}_{v}^{d}$ and $\mathfrak{X}^{d}$ be the corresponding Galton-Watson processes with offspring distributions $X_{v}$ and $X$, respectively, starting with d particles. If $X_{v} \stackrel{\mathrm{D}}{\rightarrow} X$ as $n \rightarrow \infty$, where $\stackrel{\text { ' }}{\rightarrow}$ 'denotes convergence in distribution, and $\mathrm{P}(X=1)<1$, then $\rho\left(\mathfrak{X}_{v}^{d}\right) \rightarrow \rho\left(\mathfrak{X}^{d}\right)$ for every fixed $d \geq 0$.

Proof. By (4.1), it suffices to show this for $d=1$, in which case we drop the superscript 1.

Consider the probability generating functions $f_{X}(t):=\mathrm{E} t^{X}$ and $f_{X_{v}}(t):=\mathrm{E} t^{X_{v}}$ for $0 \leq t \leq 1$. It is well known (see, e.g. [3, Theorem I.5.1]) that the extinction probability $q:=1-\rho(\mathfrak{X})$ is the smallest root in $[0,1]$ of $f_{X}(q)=q$. It follows easily, since we have excluded the possibility that $f_{X}(t) \equiv t$, that if $0 \leq t<q$ then $f_{X}(t)>t$, and if $q<t<1$ then $f_{X}(t)<t$.

Since $X_{v} \stackrel{\mathrm{D}}{\rightarrow} X$, we have $f_{X_{v}}(t) \rightarrow f_{X}(t)$ for every $t \in[0,1]$. Hence, if $0 \leq t<q$ then $f_{X_{v}}(t)>t$ for large $n$, and thus $q_{v}:=1-\rho\left(\mathfrak{X}_{v}\right)>t$. Similarly, if $q<t<1$ then, for large $n, f_{X_{v}}(t)<t$ and thus $q_{v}<t$. It follows that $q_{v} \rightarrow q$ as $n \rightarrow \infty$.

Remark 4.1. The case in which $\mathrm{P}(X=1)=1$, i.e. $X=1$ a.s., really is an exception. If we let $X_{v} \sim \operatorname{Be}\left(1-v^{-1}\right)$, we have $X_{v} \stackrel{\mathrm{D}}{\rightarrow} X=1$, but $\rho\left(\mathfrak{X}_{v}\right)=0$ for every $v$ while $\rho(\mathfrak{X})=1$.

\section{The giant component}

Our ultimate goal is to describe the large component or components of $G^{*}\left(n,\left(d_{i}\right)_{1}^{n}\right)_{\mathrm{v} ; p}$ and $G\left(n,\left(d_{i}\right)_{1}^{n}\right)_{\mathrm{v} ; p}$, where $\mathrm{v}$ is one of the vaccination strategies defined above. The basic strategy will be to relate the neighbourhoods of a vertex to a branching process. We do this for $G^{*}\left(n,\left(d_{i}\right)_{1}^{n}\right)$, which is technically easier to handle; as explained in Section 5.2, the results then transfer to $G\left(n,\left(d_{i}\right)_{1}^{n}\right)$ too, provided Condition 2.2 holds. First we argue in detail for the simplest case, namely, $G^{*}\left(n,\left(d_{i}\right)_{1}^{n}\right)$ without edge deletion (i.e. $p=1$ ) or vaccination, and prove our main results concerning the existence, size, and uniqueness of the giant component. We use and adapt the method in [5] (for a different random graph model). This will provide a new proof of the results by Molloy and Reed [15], [16] (under our slightly weaker condition). We will then describe the modifications required to make the results also valid when there is edge deletion or vaccination.

We say that an event holds with high probability (w.h.p.), if it holds with probability tending to 1 as $n$ tends to $\infty$. We shall use $o_{p}$ in the standard way (see, e.g. [11]); for example, if $\left(X_{n}\right)$ is a sequence of random variables then $X_{n}=o_{p}(1)$ means that $X_{n} \stackrel{P}{\rightarrow} 0$. We shall often use the basic fact that if $a \in \mathbb{R}$ then $X_{n} \stackrel{\mathrm{P}}{\rightarrow} a$ if and only if, for every $\varepsilon>0$, the relations $X_{n}>a-\varepsilon$ and $X_{n}<a+\varepsilon$ hold w.h.p. All unspecified limits are taken as $n$ tends to $\infty$, while $p$ and the vaccination parameters, $v$ or $c$, are kept fixed.

We denote the orders of the components of a graph $G$ by $C_{1}(G) \geq C_{2}(G) \geq \cdots$, with $C_{j}(G)=0$ if $G$ has fewer than $j$ components. We let $N_{k}(G)$ denote the total number of vertices in components of order $k$, and write $N_{\geq k}(G)$ for $\sum_{j \geq k} N_{j}(G)$, the number of vertices in components of order at least $k$. Similarly, we let $N_{k, d}(G)$ and $N_{\geq k, d}(G)$ denote the number of such vertices that have degree $d$. 
Remark 5.1. Our results are typically of the form $C_{1}\left(G_{n}\right)=\tau n+o_{p}(n)$ and $C_{\mathrm{P}}\left(G_{n}\right)=o_{p}(n)$ for some number $\tau \geq 0$ (or, equivalently, $C_{1}\left(G_{n}\right) / n \stackrel{\mathrm{P}}{\rightarrow} \tau$ and $C_{2}\left(G_{n}\right) / n \stackrel{\mathrm{P}}{\rightarrow} 0$ ). Hence, if $\tau>0$ then there exists exactly one 'giant' component, and all other components are much smaller. In our epidemic setting, this means that if $\tau=0$ then every epidemic will be 'small', i.e. $o(n)$, while if $\tau>0$ then the epidemic will be large with probability $\tau$ (allowing for the case in which the initially infected person is vaccinated and thus never becomes ill), and in this case a fraction $\tau$ of the population will be infected. ( $\tau$ thus has a double role.)

\section{1. $G^{*}\left(n,\left(d_{i}\right)_{1}^{n}\right)$, with $p=1$ and no vaccination}

As stated above, we will use a branching process approximation. The particles in the branching process correspond to free (not yet paired) half edges. Note that there are $j n_{j}$ half edges belonging to vertices of degree $j$. Hence, a random half edge shares a vertex with $j-1$ other half edges with probability $j n_{j} / \sum_{k} k n_{k}$. By Condition 2.1, jn $n_{j} / \sum_{k} k n_{k} \rightarrow j p_{j} / \mu$, and recall the definition of $\tilde{p}_{j}=j p_{j} / \mu$ defined in (3.1). Let $\mathfrak{X}$ be the Galton-Watson branching process starting with one particle and with the offsping distribution $\left(\tilde{p}_{j+1}\right)_{j=0}^{\infty}$. (This is the distribution $\left(p_{j}\right)_{j}$, size biased and shifted one step.) In other words, the offspring distribution is $\tilde{D}-1$, with $\tilde{D}$ as given in Section 3 .

We let $\rho=\rho(\mathfrak{X})$ denote the survival probability of $\mathfrak{X}$, and define

$$
\tau:=\sum_{d=1}^{\infty} p_{d}\left(1-(1-\rho)^{d}\right) ;
$$

this is the survival probability for the branching process $\mathfrak{X}$ started with a random number of particles having distribution $\left(p_{d}\right)_{d=0}^{\infty}$.

Consider a vertex $x$ of degree $d$ in $G^{*}\left(n,\left(d_{i}\right)_{1}^{n}\right)$. We explore the component containing $x$ by a breadth-first search. We concentrate on the half edges, so we begin by taking the $d$ half edges at $x$, and label them as active. We then process the active half edges one by one as follows. We take an active half edge, relabel it as used, and find the half edge that it connects to and the corresponding vertex; this partner is chosen uniformly among all half edges that are not yet used. We then label the partner as used and all other half edges at the same vertex as active, provided that they are not already used (which would mean that we have found a cycle or a multiple edge). The active half edges will behave essentially as a Galton-Watson process (where we reveal the children of the particles one by one), but the probability distribution of the children will vary slightly; it will depend on the numbers of vertices of different degrees that we have already found. Nevertheless, it is obvious that at each step in the beginning, the probability of $j-1$ new half edges is close to $j n_{j} / \sum_{k} k n_{k} \approx \tilde{p}_{j}$.

To be more precise, first, let $k$ be a fixed number, and consider the event that $x$ belongs to a component with at least $k$ vertices. This is almost the same as the probability that we will find at least $k-1$ active half edges in the process just described. (This is not exact, because if we stop when we have found $k-1$ half edges, some of these may connect back to vertices already found; however, the probability of this tends to 0 as $n$ tends to $\infty$.) The complementary event, that the process finds less than $k-1$ active half edges, consists of a finite number of cases, where each case describes the sequence of new active half edges found at each step. It is obvious that the probability of each of these cases converges, as $n$ tends to $\infty$, to the corresponding probability in $\mathfrak{X}^{d}$, and thus we find, for a vertex $x$ of degree $d$, with $\mathcal{C}(x)$ denoting the corresponding component of $G^{*}\left(n,\left(d_{i}\right)_{1}^{n}\right)$,

$$
\mathrm{P}(|\mathcal{C}(x)| \geq k)=\mathrm{P}\left(\left|\mathfrak{X}^{d}\right| \geq k-1\right)+o(1) .
$$


Recall that $N_{\geq k}$ is the number of vertices of degree $d$ belonging to a component of size at least $k$. The expectation $\mathrm{E} N_{\geq k, d}$ equals $n_{d}$ times the probability that a given vertex $x$ of degree $d$ satisfies $|\mathcal{C}(x)| \geq k$, and thus, by (5.1) and Condition 2.1(i), for every fixed $d \geq 0$ and $k \geq 1$,

$$
\mathrm{E}\left(\frac{N_{\geq k, d}}{n}\right) \rightarrow p_{d} \mathrm{P}\left(\left|\mathfrak{X}^{d}\right| \geq k-1\right) .
$$

Next we want to let $k$ tend to $\infty$ here. Thus, for the remainder of this section, we assume that $\omega(n)$ is a function such that $\omega(n) \rightarrow \infty$ but $\omega(n) / n \rightarrow 0$ as $n \rightarrow \infty$. We regard components as big if they contain at least $\omega(n)$ vertices, and small otherwise. (The flexibility in the choice of $\omega(n)$ is useful, but we will see that it does not matter much; the asymptotics we find do not depend on $\omega$.)

Lemma 5.1. If $\omega(n) \rightarrow \infty$ and $\omega(n) / n \rightarrow 0$ then,

$$
\mathrm{E}\left(\frac{N_{\geq \omega(n)}}{n}\right) \rightarrow \tau
$$

and, for every fixed $d \geq 0$,

$$
\mathrm{E}\left(\frac{N_{\geq \omega(n), d}}{n}\right) \rightarrow p_{d} \mathrm{P}\left(\left|\mathfrak{X}^{d}\right|=\infty\right)=p_{d}\left(1-(1-\rho)^{d}\right) .
$$

Proof. We begin with an upper bound in (5.4). For any fixed $k$, we have $\omega(n)>k$ for large $n$, and thus $N_{\geq \omega(n), d} \leq N_{\geq k, d}$. Consequently, (5.2) yields

$$
\limsup _{n \rightarrow \infty} \mathrm{E}\left(\frac{N_{\geq \omega(n), d}}{n}\right) \leq \limsup _{n \rightarrow \infty} \mathrm{E}\left(\frac{N_{\geq k, d}}{n}\right)=p_{d} \mathrm{P}\left(\left|\mathfrak{X}^{d}\right| \geq k-1\right) .
$$

As $k$ tends to $\infty$, the right-hand side converges to $p_{d} \mathrm{P}\left(\left|\mathfrak{X}^{d}\right|=\infty\right)$, and we find

$$
\limsup _{n \rightarrow \infty} \mathrm{E}\left(\frac{N_{\geq \omega(n), d}}{n}\right) \leq p_{d} \mathrm{P}\left(\left|\mathfrak{X}^{d}\right|=\infty\right) .
$$

For a lower bound, let $v \geq 1$ be fixed, and let $X_{v}$ be a random variable taking values in $\{0,1, \ldots, v\}$ with $\mathrm{P}\left(X_{v}=j\right)=\left(1-v^{-1}\right) \tilde{p}_{j+1}$ for $1 \leq j \leq v$ (and a suitable value for $\mathrm{P}\left(X_{v}=0\right)$ so that the sum becomes 1$)$. Consider the breadth-first exploration process described above. As long as we have found less than $\omega(n)$ vertices, the number of new active half edges at each step stochastically dominates $X_{v}$, provided $n$ is large enough, since the remaining number of vertices of degree $j+1$ is $n_{j+1}-o(n)=p_{j+1} n-o(n) \geq\left(1-v^{-1}\right) p_{j+1} n$ for large $n$. (If $p_{j+1}=0$, the result is trivial.) Consequently, letting $\mathfrak{X}_{v}^{d}$ be the Galton-Watson process with $d$ initial particles and the number of children distributed as $X_{v}$, if $n$ is large enough, we can couple the exploration process and $\mathfrak{X}_{v}^{d}$ such that as long as we have found less than $\omega(n)$ vertices, the number of active half edges is at least equal to the number of active particles in $\mathfrak{X}_{v}^{d}$ (i.e. the particles whose children have not yet been revealed). In particular, if the exploration process stops before $\omega(n)$ vertices are found then $\mathfrak{X}_{v}^{d}$ stops and, thus, the probability that a vertex $x$ of degree $d$ satisfies $|\mathcal{C}(x)|<\omega(n)$ is at most $\mathrm{P}\left(\left|\mathfrak{X}_{v}^{d}\right|<\infty\right)$. Consequently, for large $n$,

$$
\mathrm{E} N_{\geq \omega(n), d} \geq n_{d} \mathrm{P}\left(\left|\mathfrak{X}_{v}^{d}\right|=\infty\right) \text {, }
$$

and thus

$$
\liminf _{n \rightarrow \infty} \mathrm{E}\left(\frac{N_{\geq \omega(n), d}}{n}\right) \geq p_{d} \mathrm{P}\left(\left|\mathfrak{X}_{v}^{d}\right|=\infty\right)
$$


Now let $v$ tend to $\infty$. Then $X_{v} \stackrel{\mathrm{D}}{\rightarrow} X$, where $X$ has the distribution $\mathrm{P}(X=j)=\tilde{p}_{j+1}$, and thus, by Lemma 4.1, $\mathrm{P}\left(\left|\mathfrak{X}_{v}^{d}\right|=\infty\right) \rightarrow \mathrm{P}\left(\left|\mathfrak{X}^{d}\right|=\infty\right)$. Consequently,

$$
\liminf _{n \rightarrow \infty} \mathrm{E}\left(\frac{N_{\geq \omega(n), d}}{n}\right) \geq p_{d} \mathrm{P}\left(\left|\mathfrak{X}^{d}\right|=\infty\right),
$$

which together with (5.6) and (4.1) yields (5.4).

Finally, noting that $N_{\geq \omega(n), d} \leq n_{d}$, it easily follows, from the uniform summability in (2.1), that we can sum (5.4) over $d$ and take the limit outside the sum, i.e.

$$
\mathrm{E}\left(\frac{N_{\geq \omega(n)}}{n}\right)=\sum_{d} \mathrm{E}\left(\frac{N_{\geq \omega(n), d}}{n}\right) \rightarrow \sum_{d} p_{d}\left(1-(1-\rho)^{d}\right)=\tau .
$$

Note that the limits do not depend on the choice of $\omega(n)$. Hence, it follows that the expected number of vertices belonging to components of size between, say, $\log n$ and $n^{0.99}$ is $o(n)$.

We next show that we have convergence not only of the expectations but also of the random variables in (5.3) and (5.4), i.e. that these random variables are concentrated close to their expectations.

Lemma 5.2. If $\omega(n) \rightarrow \infty$ and $\omega(n) / n \rightarrow 0$ then,

$$
\frac{N_{\geq \omega(n)}}{n} \stackrel{\mathrm{P}}{\rightarrow} \tau
$$

and, for every fixed $d \geq 0$,

$$
\frac{N_{\geq \omega(n), d}}{n} \stackrel{\mathrm{P}}{\rightarrow} p_{d}\left(1-(1-\rho)^{d}\right) .
$$

Proof. Start with two distinct vertices $x$ and $z$ of the same degree $d$ and explore their components as above. We can repeat the arguments above, and find that

$$
\mathrm{P}(|\mathcal{C}(x)|<k,|\mathcal{C}(y)|<k)=\mathrm{P}\left(\left|\mathfrak{X}^{d}\right|<k-1\right)^{2}+o(1),
$$

and thus, using (5.1),

$$
\mathrm{P}(|\mathcal{C}(x)| \geq k,|\mathcal{C}(y)| \geq k)=\mathrm{P}\left(\left|\mathfrak{X}^{d}\right| \geq k-1\right)^{2}+o(1) .
$$

Multiplying by the number $n_{d}\left(n_{d}-1\right)$ of pairs $(x, y)$ of the same degree $d$, and noting that the number of such pairs, where both $x$ and $z$ belong to components of size greater than or equal to $k$ (the same or not), is $N_{\geq k, d}\left(N_{\geq k, d}-1\right)$, we find that

$$
\mathrm{E}\left(\frac{N_{\geq k, d}^{2}}{n^{2}}\right)=\mathrm{E}\left(\frac{N_{\geq k, d}\left(N_{\geq k, d}-1\right)}{n^{2}}\right)+O\left(\frac{1}{n}\right) \rightarrow p_{d}^{2} \mathrm{P}\left(\left|\mathfrak{X}^{d}\right| \geq k-1\right)^{2} .
$$

Hence, $\lim \sup _{n \rightarrow \infty} \mathrm{E}\left(N_{\geq \omega(n), d}^{2} / n^{2}\right) \leq p_{d}^{2} \mathrm{P}\left(\left|\mathfrak{X}^{d}\right| \geq k-1\right)^{2}$ for every $k$, and thus

$$
\limsup _{n \rightarrow \infty} \mathrm{E}\left(\frac{N_{\geq \omega(n), d}^{2}}{n^{2}}\right) \leq p_{d}^{2} \mathrm{P}\left(\left|\mathfrak{X}^{d}\right|=\infty\right)^{2} .
$$

Since, by the Cauchy-Schwarz inequality and (5.4),

$$
\mathrm{E}\left(\frac{N_{\geq \omega(n), d}^{2}}{n^{2}}\right) \geq\left(\mathrm{E}\left(\frac{N_{\geq \omega(n), d}}{n}\right)\right)^{2} \rightarrow p_{d}^{2} \mathrm{P}\left(\left|\mathfrak{X}^{d}\right|=\infty\right)^{2},
$$


it follows that

$$
\mathrm{E}\left(\frac{N_{\geq \omega(n), d}^{2}}{n^{2}}\right) \rightarrow p_{d}^{2} \mathrm{P}\left(\left|\mathfrak{X}_{v}^{d}\right|=\infty\right)^{2}
$$

This and (5.4) show that

$$
\operatorname{var}\left(\frac{N_{\geq \omega(n), d}}{n}\right) \rightarrow 0,
$$

and thus

$$
\frac{N_{\geq \omega(n), d}-\mathrm{E}\left(N_{\geq \omega(n), d}\right)}{n} \stackrel{\mathrm{P}}{\rightarrow} 0,
$$

which by (5.4) implies (5.8).

Finally, again we can sum over $d$ because of (2.1); this yields (5.7).

Theorem 5.1. Assume that Condition 2.1 holds. Then

$$
\begin{gathered}
C_{1}\left(G^{*}\left(n,\left(d_{i}\right)_{1}^{n}\right)\right)=\tau n+o_{p}(n), \\
C_{2}\left(G^{*}\left(n,\left(d_{i}\right)_{1}^{n}\right)\right)=o_{p}(n) .
\end{gathered}
$$

Proof. We have already shown that roughly $\tau n$ vertices lie in big components. It remains to show that most of them belong to the same component. We write $G_{n}=G^{*}\left(n,\left(d_{i}\right)_{1}^{n}\right)$.

First, if $C_{1}\left(G_{n}\right) \geq \omega(n)$ then $N_{\geq \omega(n)}\left(G_{n}\right) \geq C_{1}\left(G_{n}\right)$. Thus, for every $\varepsilon>0$ and $n$ so large that $\omega(n)<\varepsilon n$, we have, by Lemma 5.2,

$$
\mathrm{P}\left(C_{1}\left(G_{n}\right)>\tau n+\varepsilon n\right) \leq \mathrm{P}\left(N_{\geq \omega(n)}\left(G_{n}\right)>\tau n+\varepsilon n\right) \rightarrow 0 .
$$

This completes the proof if $\tau=0$.

In the sequel we assume that $\tau>0$ and show a corresponding estimate from below. First, if $p_{d}=0$ for every $d \geq 2$ then $\tilde{p}_{j+1}=0$ for all $j \geq 1$, so $\mathfrak{X}$ dies immediately and $\rho=0$ and $\tau=0$. Hence, $p_{d}>0$ for some $d \geq 2$. We fix such a $d$ for the remainder of the proof, and fix $\delta$ with $0<\delta<\frac{1}{2}$. Furthermore, take (rather arbitrarily) $\omega(n)=n^{0.9}$.

In the sequel we assume that $n$ is so large that $n_{d}>n^{1-\delta}$. We then split the first $n^{1-\delta}$ vertices of degree $d$ in $G_{n}$ into $d$ vertices of degree 1 each; we colour these $d n^{1-\delta}$ new vertices red. (To be precise, we should round $n^{1-\delta}$ to an integer.) We denote the resulting graph by $G_{n}^{\prime}$; note that $G_{n}^{\prime}$ is a random multigraph $G^{*}\left(n^{\prime},\left(d_{i}^{\prime}\right)\right)$, where $n_{j}^{\prime}$, the number of vertices of degree $j$, is given by $n_{d}^{\prime}=n_{d}-n^{1-\delta}, n_{1}^{\prime}=n_{1}+d n^{1-\delta}$, and $n_{j}^{\prime}=n_{j}$ for $j \neq 1, d$. Note that the total number of vertices in $G_{n}^{\prime}$ is $n^{\prime}:=n+(d-1) n^{1-\delta}=n+o(n)$, and that $\left(d_{i}^{\prime}\right)$ satisfies Condition 2.1 with the same $\left(p_{j}\right)$ (except that $n$ is replaced by $n^{\prime}$, which only makes a notational difference). Consequently, our results above apply to $G_{n}^{\prime}$ too.

By symmetry we may assume that the $d n^{1-\delta}$ red vertices in $G_{n}^{\prime}$ are chosen at random among all vertices of degree 1 , and that $G_{n}$ is obtained by partitioning the red vertices at random into groups with $d$ vertices and then coalescing each group into one vertex.

During the exploration of the component $\mathcal{C}^{\prime}(x)$ in $G_{n}^{\prime}$ containing a vertex $x$, in each step, the active half edge is paired with the single half edge leading to a red vertex with probability at least $c_{1} n^{-\delta}$ for some $c_{1}>0$, unless at least $n^{1-\delta}$ red vertices have already been found. Consequently, if the component $\mathcal{C}^{\prime}(x)$ has at least $\omega(n)$ vertices, the number of red vertices stochastically dominates $\min \left(n^{1-\delta}, \operatorname{Bi}\left(\omega(n)-1, c_{1} n^{-\delta}\right)\right)$. A Chernoff bound (see, e.g. [11, Corollary 2.3]) shows that the probability that $\mathcal{C}^{\prime}(x)$ has at least $\omega(n)$ vertices but less than $c_{2} n^{-\delta} \omega(n)=c_{2} n^{0.9-\delta}$ red vertices is at most $\exp \left(-c_{3} n^{0.9-\delta}\right)=o\left(n^{-1}\right)$ for $c_{2}=c_{1} / 2$ and 
some $c_{3}>0$. Summing over all $x$, we see that, w.h.p., every big component of $G_{n}^{\prime}$ contains at least $c_{2} n^{0.9-\delta}$ red vertices.

Assume that this holds, and consider two big components $K_{1}$ and $K_{2}$ in $G_{n}^{\prime}$. We can construct the random partition of the red vertices by first taking the red vertices in $K_{1}$ one by one, unless already used, and randomly selecting $d-1$ partners. We thus do this at least $m:=c_{2} n^{0.9-\delta} / d$ times, and each time the probability of not including a red vertex in $K_{2}$ is at most $1-c_{2} n^{0.9-\delta} / d n^{1-\delta}=1-c_{4} n^{-0.1}$, with $c_{4}=c_{2} / d$. Consequently, the probability of not joining $K_{1}$ and $K_{2}$ in the coalescing phase is at most

$$
\exp \left(-m c_{2} n^{-0.1}\right)=\exp \left(-c_{4}^{2} n^{0.8-\delta}\right)=o\left(n^{-2}\right) .
$$

Since there are at most $\left(n^{\prime}\right)^{2}=O\left(n^{2}\right)$ such pairs $K_{1}$ and $K_{2}$, we see that, w.h.p., all big components in $G_{n}^{\prime}$ are connected in $G_{n}$. Hence, if $B^{\prime}$ is the union of all the big components in $G_{n}^{\prime}$, and $B$ is the corresponding set of vertices in $G_{n}$, we see that, w.h.p., $B$ is connected in $G_{n}$, and, using Lemma 5.2 for $G_{n}^{\prime}$,

$$
C_{1}\left(G_{n}\right) \geq|B| \geq\left|B^{\prime}\right|-(d-1) n^{1-\delta}=\tau n^{\prime}+o_{p}(n)=\tau n+o_{p}(n) .
$$

Combining (5.10) and (5.9), we obtain $C_{1}\left(G_{n}\right)=\tau n+o_{p}(n)$.

Finally, we observe that if $C_{2}\left(G_{n}\right) \geq \omega(n)$ then $N_{\geq \omega(n)}\left(G_{n}\right) \geq C_{1}\left(G_{n}\right)+C_{2}\left(G_{n}\right)$, and thus, by (5.7) and (5.10),

$$
C_{2}\left(G_{n}\right) \leq \max \left(\omega(n), N_{\geq \omega(n)}\left(G_{n}\right)-C_{1}\left(G_{n}\right)\right)=o_{p}(n) .
$$

\subsection{The simple random graph $G\left(n,\left(d_{i}\right)_{1}^{n}\right)$}

We transfer the results to the simple random graph $G\left(n,\left(d_{i}\right)_{1}^{n}\right)$ by the following result proved in [9]; see also, e.g. [4, Section II.4] and [14] for earlier versions.

Lemma 5.3. If Conditions 2.1 and 2.2 hold then

$$
\liminf _{n \rightarrow \infty} \mathrm{P}\left(G^{*}\left(n,\left(d_{i}\right)_{1}^{n}\right) \text { is a simple graph }\right)>0 .
$$

All results for $G^{*}\left(n,\left(d_{i}\right)_{1}^{n}\right)$ that can be stated in terms of convergence in probability, as our results in this section, thus hold also if we condition on the graph being simple. In other words, the results proved for $G^{*}\left(n,\left(d_{i}\right)_{1}^{n}\right)$ hold for $G\left(n,\left(d_{i}\right)_{1}^{n}\right)$ too. Thus, Theorem 5.1 has the following version for $G\left(n,\left(d_{i}\right)_{1}^{n}\right)$.

Theorem 5.2. Assume that Conditions 2.1 and 2.2 hold. Then

$$
\begin{gathered}
C_{1}\left(G\left(n,\left(d_{i}\right)_{1}^{n}\right)\right)=\tau n+o_{p}(n), \\
C_{2}\left(G\left(n,\left(d_{i}\right)_{1}^{n}\right)\right)=o_{p}(n) .
\end{gathered}
$$

\subsection{Uniform vaccination}

We now extend Theorem 5.1 to the graph $G^{*}\left(n,\left(d_{i}\right)_{1}^{n}\right)_{v ; p}^{\mathrm{U}}$, where $0 \leq v<1$ and $0<$ $p \leq 1$; see Section 2. Recall that we obtain this graph from $G^{*}\left(n,\left(d_{i}\right)_{1}^{n}\right)$ by randomly and independently deleting edges with probability $1-p$ (nontransmission) and vertices with probability $v$ (vaccination). The branching process approximation arguments above still work, with the difference that each new individual found is kept with probability $p(1-v)$, and otherwise discarded. Hence, the offspring distribution is changed from $\tilde{D}-1$ to $\tilde{X}_{v} \sim$ $\operatorname{Mix} \operatorname{Bi}(\tilde{D}-1, p(1-v))$, and the branching process corresponding to an unvaccinated person 
with $d$ friends starts with $\operatorname{Bi}(d, p(1-v))$ individuals. Now let $\mathfrak{X}^{d}$ denote the branching process with this offspring distribution, starting with $d$ individuals. The probability generating function of $\tilde{X}_{v}$ is, as shown in Sections 3.1 and 3.2, given by

$$
\mathrm{E} t^{\tilde{X}_{v}}=\frac{f_{D}^{\prime}(1-p(1-v)(1-t))}{f_{D}^{\prime}(1)} .
$$

Hence, the extinction probability of $\mathfrak{X}^{1}$ is $\tilde{\pi}_{v ; p}^{\cup}$, given by (3.5). If we start the branching process with $D^{\prime} \sim \operatorname{Bi}(d, p(1-v))$ individuals, the extinction probability is thus, writing $\bar{p}=p(1-v)$,

$$
\pi^{(d)}:=\sum_{k}\left(\begin{array}{l}
d \\
k
\end{array}\right) \bar{p}^{k}(1-\bar{p})^{d-k}\left(\tilde{\pi}_{v ; p}^{U}\right)^{k}=\left(1-\bar{p}+\bar{p} \tilde{\pi}_{v ; p}^{U}\right)^{d} .
$$

The arguments in the proofs of Lemmas 5.1 and 5.2 show, recalling that each vertex has probability $1-v$ of being unvaccinated, that (5.8) holds in the form

$$
\frac{N_{\geq \omega(n), d}}{n} \stackrel{\mathrm{P}}{\rightarrow} p_{d}(1-v)\left(1-\pi^{(d)}\right)
$$

for every fixed $d \geq 0$, assuming that $\omega(n) \rightarrow \infty$ and $\omega(n) / n \rightarrow 0$. Hence,

$$
\frac{N_{\geq \omega(n)}}{n(1-v)} \stackrel{\mathrm{P}}{\rightarrow} \sum_{d} p_{d}\left(1-\pi^{(d)}\right)=1-\sum_{d} p_{d} \pi^{(d)}=1-f_{D}\left(1-\bar{p}+\bar{p} \tilde{\pi}_{v ; p}^{\mathrm{U}}\right) .
$$

This limit equals $\tau_{v ; p}^{\cup}:=1-\pi_{v ; p}^{\cup}$, with $\pi_{v ; p}^{\cup}$ given by (3.6).

To extend Theorem 5.1, it remains to show that there is only one very large component. More precisely, we again show that, with $\omega(n)=n^{0.9}$, there is, w.h.p., only one big component. We argue as in Theorem 5.1, splitting some vertices of degree $d$ in $G_{n}=G^{*}\left(n,\left(d_{i}\right)_{1}^{n}\right)$ into $d$ red vertices of degree 1 , calling the resulting graph $G_{n}^{\prime}$.

We vaccinate the vertices in $G_{n}^{\prime}$ with probability $v$ each, independently; we then recombine the red vertices to vertices of degree $d$ in $G_{n}$, and consider each such vertex as vaccinated if at least one of its red parts in $G_{n}^{\prime}$ is vaccinated. This means that some vertices in $G_{n}$ are vaccinated with probability larger than $v$, but this does not matter since the aim of the argument is to provide a lower bound for $C_{1}$, the size of the largest component, and any extra vaccinations can only decrease $C_{1}$.

By a Chernoff bound there are, w.h.p., at least $(1-v) n^{1-\delta}$ unvaccinated red vertices, and as before it follows that, w.h.p., every big component of $\left(G_{n}^{\prime}\right)_{v ; p}^{\mathrm{U}}$ contains at least $c_{2} n^{0.9-\delta}$ red vertices (although the value of $c_{2}$ may change). Given two big components $K_{1}$ and $K_{2}$ it follows, similarly as before, that, with probability $1-o\left(n^{-2}\right)$, there exists a vertex in $G_{n}$ that is split into $d$ red vertices, of which at least one is in $K_{1}$, at least one is in $K_{2}$, and all are unvaccinated. The proof is completed as before.

Consequently, also using Lemma 5.3, we have the following theorem. Theorems 3.2 and 3.1 (the special case in which $v=0$ ) are immediate consequences.

Theorem 5.3. Assume that Condition 2.1 holds, and let $0<p \leq 1$ and $0 \leq v<1$. Then,

$$
\begin{gathered}
C_{1}\left(G^{*}\left(n,\left(d_{i}\right)_{1}^{n}\right)_{v ; p}^{\mathrm{U}}\right)=\tau_{v ; p}^{\mathrm{U}} n(1-v)+o_{p}(n), \\
C_{2}\left(G^{*}\left(n,\left(d_{i}\right)_{1}^{n}\right)_{v ; p}^{\mathrm{U}}\right)=o_{p}(n),
\end{gathered}
$$

where $\tau_{v ; p}^{\cup}=1-\pi_{v ; p}^{\cup}$, with $\pi_{v ; p}^{\cup}$ given by (3.6). Also if Condition 2.2 holds then the same results hold for $G\left(n,\left(d_{i}\right)_{1}^{n}\right)_{v ; p}^{\cup}$ too. 


\subsection{Acquaintance vaccination}

As explained in Section 3.3, in order to obtain (asymptotically) a Galton-Watson branching process, with the right independence properties, we consider directed edges, or equivalently half edges, that are open, i.e. transmission may take place but the edge is not used for vaccination. Moreover, we consider only open edges originating at an unvaccinated person.

Let $x$ be a given vertex with degree $d$ in $G^{*}\left(n,\left(d_{i}\right)_{1}^{n}\right)$, and let us explore the component of $x$ in $G^{*}\left(n,\left(d_{i}\right)_{1}^{n}\right)_{c ; p}^{\mathrm{A}}$, conditioned on $x$ being unvaccinated (otherwise $x$ does not belong to $\left.G^{*}\left(n,\left(d_{i}\right)_{1}^{n}\right)_{c ; p}^{\mathrm{A}}\right)$. In order to be kept in $G^{*}\left(n,\left(d_{i}\right)_{1}^{n}\right)_{c ; p}^{\mathrm{A}}$, an edge has to be open, but not all edges are kept since some may lead to vertices that are vaccinated; see Figure 1(c). Nevertheless, we consider all open edges found during the exploration. We declare the open edges starting at $x$ to be active. We then investigate the active edges. If an active edge leads to a person that is unvaccinated, we declare the open edges going from that person, except the one going back to where we just came from, to be new active edges. We continue until no more active edges are found; we then have found the component containing $x$ (plus some extra open edges leading to vaccinated persons).

We investigate this process probabilistically, revealing the structure of $G^{*}\left(n,\left(d_{i}\right)_{1}^{n}\right)$ by combining half edges at random during the exploration. We consider asymptotics as $n$ tends to $\infty$, and some of the statements below are only approximately correct for finite $n$.

First note that each of the $d$ edges leading from $x$ is open with probability $p \mathrm{e}^{-c / d}$, independently of each other, so we start with $\operatorname{Bi}\left(d, p \mathrm{e}^{-c / d}\right)$ open edges.

The vertex $x$ has $d$ friends; in $G^{*}\left(n,\left(d_{i}\right)_{1}^{n}\right)$ they are chosen by randomly choosing $d$ half edges and their degrees have the size-biased distribution $\left(\tilde{p}_{j}\right)$, independently of each other. Conditioning on $x$ being unvaccinated means that we condition on none of the $d$ edges being used for vaccination in the opposite direction. Since the probability that a friend with degree $j$ does not name $x$ is $\mathrm{e}^{-c / j}$, this preserves the independence of the degrees of the friends, but shifts their distribution to, as asserted in (3.10), $\left(\tilde{p}_{j} \mathrm{e}^{-c / j} / \alpha\right)_{j}$, where

$$
\alpha=\alpha(c)=\sum_{j} \tilde{p}_{j} \mathrm{e}^{-c / j},
$$

as in (3.7), is the probability of not being named by a random friend.

Now suppose that an open edge goes from $x$ to a friend $z$ of degree $k$. In order for this to define an edge in $G^{*}\left(n,\left(d_{i}\right)_{1}^{n}\right)_{c ; p}^{\mathrm{A}}, z$ must not be vaccinated through another of his or her friends; this has the probability $\alpha^{k-1}$. In this case $z$ has $k-1$ further edges, and each of them is open with probability $p \mathrm{e}^{-c / k}$. It follows that the number of new open edges at $z$ has a distribution that is the mixture $\left(1-\alpha^{k-1}\right) \delta_{0}+\alpha^{k-1} \operatorname{Bi}\left(k-1, p \mathrm{e}^{-c / k}\right)$. Using the distribution in (3.10) for the degree of $z$, we finally see that the distribution of the number $Y$ of new active edges found when exploring a single active edge is given by (3.11).

Hence, observing obvious independence properties, the process of active edges is (asymptotically) a Galton-Watson branching process with offspring distribution $Y$, starting with $\operatorname{Bi}\left(d, p \mathrm{e}^{-c / d}\right)$ active edges. Denote this branching process by $\mathfrak{X}^{(d)}$. Let, as in Section 3.3, $\tilde{\pi}_{c ; p}^{\mathrm{A}}$ be the probability that a branching process with this offspring distribution $Y$ and starting 
with a single individual dies out. Then, the extinction probability of $\mathfrak{X}^{(d)}$ is

$$
\begin{aligned}
\pi^{(d)} & :=\mathrm{P}\left(\left|\mathfrak{X}^{(d)}\right|<\infty\right) \\
& =\sum_{j=0}^{d}\left(\begin{array}{l}
d \\
j
\end{array}\right)\left(p \mathrm{e}^{-c / d}\right)^{j}\left(1-p \mathrm{e}^{-c / d}\right)^{d-j}\left(\tilde{\pi}_{c ; p}^{\mathrm{A}}\right)^{j} \\
& =\left(1-p \mathrm{e}^{-c / d}+p \mathrm{e}^{-c / d} \tilde{\pi}_{c ; p}^{\mathrm{A}}\right)^{d} .
\end{aligned}
$$

A minor complication is that the branching process approximation counts open edges and, as remarked above, not all open edges lead to vertices in $G^{*}\left(n,\left(d_{i}\right)_{1}^{n}\right)_{c ; p}^{\mathrm{A}}$. Thus, (5.1) does not extend directly. However, we still have the inequality

$$
\mathrm{P}(|\mathcal{C}(x)| \geq k) \leq \mathrm{P}\left(\left|\mathfrak{X}^{(d)}\right| \geq k-1\right)+o(1) .
$$

Furthermore, a vertex of degree $d$ in $G^{*}\left(n,\left(d_{i}\right)_{1}^{n}\right)$ is unvaccinated with probability $\alpha^{d}$, and thus

$$
\mathrm{E}\left(N_{\geq k, d}\right) \leq n_{d} \alpha^{p}\left(\mathrm{P}\left(\left|\mathfrak{X}^{(d)}\right| \geq k-1\right)+o(1)\right),
$$

which arguing as in (5.5) and (5.6) leads to

$$
\limsup _{n \rightarrow \infty} \mathrm{E}\left(\frac{N_{\geq \omega(n), d}}{n}\right) \leq p_{d} \alpha^{p} \mathrm{P}\left(\left|\mathfrak{X}^{(d)}\right|=\infty\right)=p_{d} \alpha^{p}\left(1-\pi^{(d)}\right) .
$$

For a lower bound, we note that an open edge creates new open edges in the exploration process only if it leads to an unvaccinated person. Hence, if $f\left(\mathfrak{X}^{(d)}\right)$ denotes the number of individuals in the branching process $\mathfrak{X}^{(d)}$ with at least one child, we have, for every $k \geq 1$,

$$
\mathrm{P}(|\mathcal{C}(x)| \geq k) \geq \mathrm{P}\left(f\left(\mathfrak{X}^{(d)}\right) \geq k-1\right)+o(1) .
$$

In order to replace the fixed $k$ by $\omega(n)$, we proceed as in the proof of Lemma 5.1 and define a Galton-Watson process $\mathfrak{X}_{v}^{(d)}$, now starting with $\operatorname{Bi}\left(d, p \mathrm{e}^{-c / d}\left(1-v^{-1}\right)\right)$ individuals and with an offspring distribution $Y_{v}$ on $\{0, \ldots, v\}$ with $\mathrm{P}\left(Y_{v}=j\right)=\left(1-v^{-1}\right) \mathrm{P}(Y=j)$ for $j=1, \ldots, v$.

For each $v$ and each fixed $A<\infty$, we can, for large $n$, couple the exploration process and $\mathfrak{X}_{v}^{(d)}$ as in the proof of Lemma 5.1 as long as we have found at most $A \omega(n)$ open edges. Hence, if $|\mathcal{C}(x)|<\omega(n)$ then either $f\left(\mathfrak{X}_{v}^{(d)}\right)<\omega(n)$ or the process $\mathfrak{X}_{v}^{(d)}$ reaches more than $A \omega(n)$ individuals while less than $\omega(n)$ of them, plus the root, have had children. The probability of the latter event is at most, since the root has at most $d$ children,

$$
\mathrm{P}\left(1+d+\sum_{i=1}^{\omega(n)} Y_{v, i}^{*}>A \omega(n)\right),
$$

where $Y_{v, i}^{*}$ are independent random variables with the distribution $\mathcal{L}(Y \mid Y>0)$, and thus this probability tends to 0 by the law of large numbers provided that we have chosen $A>$ $\mathrm{E}(Y \mid Y>0)$. Consequently,

$$
\mathrm{P}(|\mathcal{C}(x)|<\omega(n)) \leq \mathrm{P}\left(f\left(\mathfrak{X}_{v}^{(d)}\right)<\omega(n)\right)+o(1) \leq \mathrm{P}\left(f\left(\mathfrak{X}_{v}^{(d)}\right)<\infty\right)+o(1) .
$$

Again using the fact that a person with degree $d$ is unvaccinated with probability $\alpha^{d}$, it follows that

$$
\mathrm{E} N_{\geq \omega(n), d} \geq n_{d} \alpha^{p}\left(1-\mathrm{P}\left(\left|\mathfrak{X}_{\nu}^{(d)}\right|<\infty\right)+o(1)\right),
$$


and thus

$$
\liminf _{n \rightarrow \infty} \mathrm{E}\left(\frac{N_{\geq \omega(n), d}}{n}\right) \geq p_{d} \alpha^{p} \mathrm{P}\left(\left|\mathfrak{X}_{\nu}^{(d)}\right|=\infty\right) .
$$

We let $v$ tend to $\infty$ and obtain, by Lemma 4.1 ,

$$
\liminf _{n \rightarrow \infty} \mathrm{E}\left(\frac{N_{\geq \omega(n), d}}{n}\right) \geq p_{d} \alpha^{p} \mathrm{P}\left(\left|\mathfrak{X}^{(d)}\right|=\infty\right)=p_{d} \alpha^{p}\left(1-\pi^{(d)}\right),
$$

which together with (5.11) yields

$$
\mathrm{E}\left(\frac{N_{\geq \omega(n), d}}{n}\right) \rightarrow p_{d} \alpha^{p}\left(1-\pi^{(d)}\right)
$$

Arguing as in the proof of Lemma 5.2, we also find that

$$
\frac{N_{\geq \omega(n), d}}{n} \stackrel{\mathrm{P}}{\rightarrow} p_{d} \alpha^{p}\left(1-\pi^{(d)}\right)
$$

and, recalling (3.14) and (3.8),

$$
\frac{N_{\geq \omega(n)}}{n} \stackrel{\mathrm{P}}{\rightarrow} \sum_{d} p_{d} \alpha^{p}\left(1-\pi^{(d)}\right)=\sum_{d} p_{d} \alpha^{p}\left(1-\pi_{c ; p}^{\mathrm{A}}\right)=(1-v(c)) \tau_{c ; p}^{\mathrm{A}},
$$

with $\tau_{c ; p}^{\mathrm{A}}=1-\pi_{c ; p}^{\mathrm{A}}$. In particular,

$$
C_{1}\left(G^{*}\left(n,\left(d_{i}\right)_{1}^{n}\right)_{c ; p}^{\mathrm{A}}\right) \leq \omega(n)+N_{\geq \omega(n)} \leq(1-v(c)) \tau_{c ; p}^{\mathrm{A}} n+o_{p}(n) .
$$

Finally, we argue again as in the proof of Theorem 5.1 to show that most vertices in large components belong to a single component. We split some of the vertices in $G_{n}=G^{*}\left(n,\left(d_{i}\right)_{1}^{n}\right)$ as above and perform acquaintance vaccination on the resulting graph $G_{n}^{\prime}$. This corresponds to acquaintance vaccination on $G_{n}$, except that the vertices that are now split are asked to name a friend $\operatorname{Po}(d c)$ times instead of $\operatorname{Po}(c)$. We thus perform some extra vaccinations, but this can only decrease $C_{1}$, and we obtain, as in (5.10), the lower bound

$$
C_{1}\left(G^{*}\left(n,\left(d_{i}\right)_{1}^{n}\right)_{c ; p}^{\mathrm{A}}\right) \geq(1-v(c)) \tau_{c ; p}^{\mathrm{A}} n+o_{p}(n) .
$$

Summing up, and using Lemma 5.3, we have proved the following theorem. Theorem 3.3 is an immediate consequence.

Theorem 5.4. Assume that Condition 2.1 holds, and let $0<p \leq 1$ and $0 \leq c<\infty$. Then,

$$
\begin{gathered}
C_{1}\left(G^{*}\left(n,\left(d_{i}\right)_{1}^{n}\right)_{c ; p}^{\mathrm{A}}\right)=\tau_{c ; p}^{\mathrm{A}} n(1-v(c))+o_{p}(n), \\
C_{2}\left(G^{*}\left(n,\left(d_{i}\right)_{1}^{n}\right)_{c ; p}^{\mathrm{A}}\right)=o_{p}(n),
\end{gathered}
$$

where $\tau_{c ; p}^{\mathrm{A}}=1-\pi_{c ; p}^{\mathrm{A}}$, with $\pi_{c ; p}^{\mathrm{A}}$ given by (3.14). Also if Condition 2.2 holds then the same results hold for $G\left(n,\left(d_{i}\right)_{1}^{n}\right)_{c ; p}^{\mathrm{A}}$ too. 


\subsection{Edgewise vaccination}

We argue as for acquaintance vaccination with the modifications (simplifications) explained in Section 3.4. There are no new complications, and we obtain the following theorem. Theorem 3.4 is an immediate consequence.

Theorem 5.5. Assume that Condition 2.1 holds, and let $0<p \leq 1$ and $0<\alpha \leq 1$. Then, for $j=1,2$,

$$
\begin{gathered}
C_{1}\left(G^{*}\left(n,\left(d_{i}\right)_{1}^{n}\right)_{\alpha ; p}^{\mathrm{E}_{j}}\right)=\tau_{\alpha ; p}^{\mathrm{E}_{j}} n(1-v(\alpha))+o_{p}(n), \\
C_{2}\left(G^{*}\left(n,\left(d_{i}\right)_{1}^{n}\right)_{\alpha ; p}^{\mathrm{E}_{j}}\right)=o_{p}(n),
\end{gathered}
$$

where $\tau_{\alpha ; p}^{\mathrm{E}_{j}}=1-\pi_{\alpha ; p}^{\mathrm{E}_{j}}$, with $\pi_{\alpha ; p}^{\mathrm{Ej}_{j}}$ given by (3.17). Also if Condition 2.2 holds then the same results hold for $G\left(n,\left(d_{i}\right)_{1}^{n}\right)_{\alpha ; p}^{E_{j}}$ too.

\section{Acknowledgements}

We thank Mathias Lindholm for help in producing the figures. TB gratefully acknowledges financial support from the Swedish Research Council.

\section{References}

[1] Andersson, H. (1999). Epidemic models and social networks. Math. Scientist 24, 128-147.

[2] Andersson, H. And Britton, T. (2000). Stochastic Epidemic Models and Their Statistical Analysis (Lecture Notes Statist. 151). Springer, New York.

[3] Athreya, K. B. And Ney, P. E. (1972). Branching Processes. Springer, Berlin.

[4] Bollobás, B. (2001). Random Graphs, 2nd edn. Cambridge University Press.

[5] Bollobás, B., Janson, S. And Riordan, O. (2007). The phase transition in inhomogeneous random graphs. Random Structures Algorithms 31, 3-122.

[6] Britton, T., Deijfen, M. and Martin-Löf, A. (2006). Generating simple random graphs with prescribed degree distribution. J. Statist. Phys. 124, 1377-1397.

[7] Cohen, R. Havlin, S. and Ben-Avrahan, D. (2003). Efficient immunization strategies for computer networks and populations. Phys. Rev. Lett. 91, 247901.

[8] Gut, A. (2005). Probability: A Graduate Course. Springer, New York.

[9] JANSON, S. (2006). The probability that a random multigraph is simple. Available at http://arxiv.org/abs/math/ 0609802 .

[10] JANSON, S. (2007). Asymptotic equivalence and contiguity of some random graphs. In preparation.

[11] JANSON, S., ŁUCZAK, T. AND RucińsKi, A. (2000). Random Graphs. John Wiley, New York.

[12] Janson, S., Knuth, D., ŁuczaK, T. and Pittel, B. (1994). The birth of the giant component. Random Structures Algorithms 4, 231-358.

[13] Kallenberg, O. (2002). Foundations of Modern Probability, 2nd edn. Springer, New York.

[14] McKaY, B. D. (1985). Asymptotics for symmetric 0-1 matrices with prescribed row sums. Ars Combin. 19, 15-25.

[15] Molloy, M. ANd Reed, B. (1995). A critical point for random graphs with a given degree sequence. Random Structures Algorithms 6, 161-179.

[16] Molloy, M. AND Reed, B. (1998). The size of the giant component of a random graph with a given degree sequence. Combin. Prob. Comput. 7, 295-305.

[17] Moore, C. and Newman, M. E. J. (2000). Epidemics and percolation in small world networks. Phys. Rev. E 61, 5678-5682.

[18] Newman, M. E. J. (2003). The structure and function of complex networks. SIAM Rev. 45, 167-256.

[19] Newman, M. E. J., Strogatz, S. H. and Watts, J. (2001). Random graphs with arbitrary degree distributions and their applications. Phys. Rev. E 64, 026118.

[20] Scotт, J. (2000). Social Network Analysis, A Handbook, 2nd edn. Sage, London. 